\title{
A SLIDING MESH-MORTAR METHOD FOR A TWO DIMENSIONAL EDDY CURRENTS MODEL OF ELECTRIC ENGINES
}

\author{
Annalisa Buffa $^{1}$, Yvon Maday ${ }^{2,3}$ and Francesca Rapetti ${ }^{2}$
}

\begin{abstract}
The paper deals with the application of a non-conforming domain decomposition method to the problem of the computation of induced currents in electric engines with moving conductors. The eddy currents model is considered as a quasi-static approximation of Maxwell equations and we study its two-dimensional formulation with either the modified magnetic vector potential or the magnetic field as primary variable. Two discretizations are proposed, the first one based on curved finite elements and the second one based on iso-parametric finite elements in both the static and moving parts. The coupling is obtained by means of the mortar element method (see [7]) and the approximation on the whole domain turns out to be non-conforming. In both cases optimal error estimates are provided. Numerical tests are then proposed in the case of standard first order finite elements to test the reliability and precision of the method. An application of the method to study the influence of the free part movement on the currents distribution is also provided.
\end{abstract}

Mathematics Subject Classification. 35Q60, 65N15, 65M55, 68U20, 78A30.

Received: April 12, 2000. Revised: December, 2000.

\section{INTRODUCTION}

The computation of the space and time distribution of the induced currents in electromagnetic systems is of great importance for performances prediction and devices design. Generated in conducting materials by temporal variations of the surrounding magnetic field, the induced currents can have positive or negative effects depending on the application. In the case of the induction engines the presence of induced currents is positive and generates the propulsion forces which are at the basis of the working of the engine. This is not the case for transformers: any energy lost under heat form by Joule's effect in the ferromagnetic material during the transfer from the primary to the secondary coil, reduces the efficiency of a transformer.

The subject of our research activity is the analysis and development of simulation tools to effectively predict the induced currents distribution in non-stationary geometries with sliding interfaces. As an example, in this paper we will study a system composed of two solid parts: a fixed one (stator) and a moving one (rotor) which turns around a given axis.

Several configurations of the physical system are considered: completely conducting ones with discontinuous conductivities are analyzed as well as partially conducting ones with, for example, air-gaps or materials with different magnetic properties.

\footnotetext{
Keywords and phrases. Eddy currents problem, non-conforming finite element approximation, domain decomposition methods.

${ }^{1}$ Dipartimento di Matematica, Universitá di Pavia, Via Abbiategrasso 209, 27100 Pavia, Italy. e-mail: annalisa@dimat. unipv .it

2 Applications Scientifiques du Calcul Intensif, UPR 9029 CNRS, bâtiment 506, Université Paris XI, 91403 Orsay, France.

${ }^{3}$ Laboratoire d'Analyse Numérique, Université Paris VI, 4 place Jussieu, 75252 Paris, France.
} 
We use a two-dimensional mathematical model as it results classically in the following situations:

- The physical system can be considered as an infinitely long cylinder and the sources are taken either tangent or orthogonal to the transverse section of the physical system we are considering: this leads to two-dimensional problems which are known in literature as TE (transverse electric) and TM (transverse magnetic) formulations respectively [18]. Here we have adopted the wave propagation terminology where the propagation direction becomes the diffusion one.

- The displacement currents can be neglected with respect to the conducting ones: we solve a quasi-static approximation of Maxwell's equations which is known as eddy currents model. From the mathematical point of view we deal with a degenerate parabolic problem.

- We aim at studying the variation of the current distribution due to the rotor movement: this suggests working in the time domain instead of the frequency one.

When a part of the physical system is moving with great amplitude with respect to the rest, the use of a standard conforming Galerkin method based on the deformation of the finite elements is not recommended. Due to the elements distortion, a partial or total re-meshing of the computational domain may be necessary. Generally, the re-meshing process and, most of all, the interpolation of the unknowns at the new nodes increase the computational cost of the numerical simulations. Some techniques that minimize this cost have already been analyzed: see, for example, the macro-element method [25], the moving layer method [15], a finite elementsboundary elements coupling [22].

In this paper, we propose a non-conforming (in space) approximation of the problem based on the so called mortar element method (see [7] and [4]). This method allows for non-matching grids at the interface between the stator and rotor domains. The matching constraint at the interface between the rotor and stator is weakly imposed on the discrete solution by means of Lagrange multipliers. Note that our approach differs radically from that proposed in $[21,26]$ even if the terminology is quite similar. Indeed, here, the Lagrange multiplier is never interpreted in terms of the primal unknown.

The discrete approximation space for Lagrange multipliers is chosen, according to the mortar element method theory, as the space of traces at $\Gamma$ of discrete functions in the stator part (or equivalently in the rotor one). Then the Lagrange multipliers are eliminated and we propose to solve the primal problem (and not a mixed one) in a constrained space which turns out to be a non-conforming approximation of the continuous functional framework (see [7] or [4] for details).

The use of a method with non-matching grids allows us to work with only one whole mesh composed of a fixed part and a rotating one: no heavy constraints are imposed between the discretization parameter $h$ (that is the maximum of the mesh elements diameters) at the interface and the rotation angle associated at each time step. Loosely speaking, the two discretizations of the interface induced by the stator and the rotor meshes do not need to coincide after each discrete rotation angle of the rotor domain as in the lock-step method [16].

Note that the main purpose of this paper is to propose a new non-conforming spatial approximation and as far as the time discretization is concerned, we adopt here an implicit Euler scheme as an "example" of time stepping. Thanks to the flexibility in the way the movement is treated (no constraint between time step, mesh size and the speed of the rotor), we expect that any discretization scheme can be used in time providing an optimal rate of convergence [27].

For what concerns the organization of the paper, in Section 1 we deduce the two-dimensional model from the system of Maxwell's equations, we write the variational formulation and prove the well posedness of the continuous problem. In Section 2 we propose the spatial discretization based on the mortar element method and in Section 3 we present the time discretization, providing a complete analysis of the method convergence as well as an optimal error estimate. In Section 4 the effect of iso-parametric finite elements at the interface is studied and optimal error estimates are provided also in this case. In Section 5 we detail the implementation aspects of the proposed method in case of standard linear finite elements resulting in the solution of a symmetric and positive definite system. Finally, Section 6 is dedicated to some numerical results both of validation and application of the method to an academic concrete problem; we refer to [24] for more physical examples. 


\section{The Continuous problem}

For the sake of completeness, we briefly deduce the mathematical models of the problem we are going to deal with, starting from the system of Maxwell's equations and under classical assumptions. In this short presentation, we will focus our attention only on the derivation of the equations and not on the initial and boundary conditions that we suppose equal to zero (see [14], Vol. 1 for more general situations).

Let $\mathcal{D}$ be an infinitely long cylinder in $\mathbb{R}^{3}$ with a Lipschitz transverse section $\Omega$. We fix a system of coordinates $(x, y, z)$ such that $(x, y)$ are the transverse ones.

In the following we will use the bold style to denote three-dimensional vectors and the Roman style for scalars. The system of Maxwell equations in $\mathcal{D}$, when the displacement currents are neglected, reads as follows (see [8], Chap. 4):

$\begin{array}{lll}\text { (a) } & \boldsymbol{\nabla} \times \mathbf{E}=-\frac{\partial \mathbf{B}}{\partial t} & \text { in } \mathcal{D} \\ (b) & \boldsymbol{\nabla} \times \mathbf{H}=\sigma \mathbf{E}+\mathbf{J}_{s} & \text { in } \mathcal{D} \\ (c) & \nabla \cdot(\varepsilon \mathbf{E})=0 & \text { where } \sigma=0 \\ (d) & \mathbf{B}=\mu \mathbf{H} & \text { in } \mathcal{D} \\ (e) & \text { initial conditions } & \text { in } \mathcal{D} \\ (f) & \text { boundary conditions } & \text { on } \partial \mathcal{D}\end{array}$

where $\mathbf{E}, \mathbf{H}, \mathbf{B}$ are the electric, the magnetic fields and the magnetic induction respectively.

Concerning the magnetic permeability $\mu$, the electric permittivity $\varepsilon$ and the electric conductivity $\sigma$, we assume that the material is homogeneous and isotropic in the two transverse directions. Hence,

- they are functions, not tensors;

- they respect the symmetry of the domain, in the sense that

$$
\varepsilon(x, y, z)=\varepsilon(x, y), \quad \mu(x, y, z)=\mu(x, y), \quad \sigma(x, y, z)=\sigma(x, y) .
$$

Both $\mu$ and $\sigma$ are completely characterized by $\mu_{\mid \Omega}$ and $\sigma_{\mid \Omega}$.

- We assume that $\mu \in L^{\infty}(\Omega)$ and that there exists a $\bar{\mu}$ such that $\mu(\mathbf{x}) \geq \bar{\mu}>0$ a.e. $\mathbf{x} \in \Omega$. For the conductivity, we suppose that $\sigma \in L^{\infty}(\Omega)$ and $\sigma(\mathbf{x}) \geq 0$ a.e. $\mathbf{x} \in \Omega$. ¿From now on, we denote by $\mathcal{C}$ the conducting part of $\Omega($ i.e. $\mathcal{C}:=\operatorname{supp}\{\sigma\})$ and by $\mathcal{I}:=\Omega \backslash \operatorname{supp}\{\sigma\}$ the non-conducting part, that may be reduced to the empty set.

Moreover we assume that the system (1) admits a unique solution (E, H, B). It is well known (see [20]) that it is true under some regularity assumptions on the parameters and on the transverse section $\Omega$ and when suitable boundary conditions are chosen.

In case the provided source $\mathbf{J}_{s}$ respects also the symmetry of the domain, the system (1) can be reduced to a scalar equation. Basically two different situations may occur [2].

\subsection{Magnetic equation for the TE formulation}

We consider here a system totally made of conducting material $(\mathcal{I}=\emptyset)$ and, moreover we assume that the given current density verifies:

$$
\mathbf{J}_{s}=\left(\mathrm{J}_{x}(x, y, t), \mathrm{J}_{y}(x, y, t), 0\right) \text { and } \nabla \cdot\left(\mathbf{J}_{s}\right)=0 .
$$

From equation (a) in (1) and the uniqueness of the solution of the system (1), we deduce that the electric and magnetic fields take the form

$$
\mathbf{E}=\left(\mathrm{E}_{x}(x, y, t), \mathrm{E}_{y}(x, y, t), 0\right), \quad \mathbf{H}=(0,0, \mathrm{H}(x, y, t))
$$


Then, by eliminating the electric field using equations $(a),(b)$ and $(d)$ in $(1)$, we obtain the following scalar equation for the third component $\mathrm{H}$ of the magnetic field $\mathbf{H}$ :

$$
\mu \frac{\partial \mathrm{H}}{\partial t}-\nabla \cdot\left(\sigma^{-1} \nabla \mathrm{H}\right)=\nabla \times\left(\sigma^{-1} \mathrm{~J}_{x}, \sigma^{-1} \mathrm{~J}_{y}\right)
$$

where $\nabla \times\left(a_{x}, a_{y}\right)$ is the scalar defined as

$$
\nabla \times\left(a_{x}, a_{y}\right)=\left(\frac{\partial a_{x}}{\partial y}-\frac{\partial a_{y}}{\partial x}\right)
$$

By solving equation (2) and using equations $(b)$ and $(d)$ of $(1)$ we can recover both the vectorial electric field and the scalar magnetic induction.

\subsection{Modified magnetic potential equation for the TM formulation}

When the current density $\mathbf{J}_{s}$ has the form:

$$
\mathbf{J}_{s}=\left(0,0, \mathrm{~J}_{s}(x, y, t)\right)
$$

the system (1) can again be reduced to a scalar partial differential equation. This equation can be used to compute both the electric field [10] and the so-called modified vector potential [11]. We derive here the equation for the modified vector potential. From which, both magnetic field (computing the curl) and the electric field (computing the time derivative) can be easily obtained. From equation $(b)$ in $(1)$ and the uniqueness of the solution of system (1), we have that the electric and magnetic fields take the form:

$$
\mathbf{H}=\left(\mathrm{H}_{x}(x, y, t), \mathrm{H}_{y}(x, y, t), 0\right), \quad \mathbf{E}=(0,0, \mathrm{E}(x, y, t)) .
$$

From $(a)$ in (1), we introduce the quantity

$$
\mathrm{A}(x, y, t)=-\int_{0}^{t} \mathrm{E}(x, y, s) \mathrm{d} s
$$

which is the third component, and the only non-zero one, of the modified magnetic vector potential. By eliminating the magnetic field $\mathbf{H}$ from equations $(a)$ and $(b)$ in (1), we obtain a scalar equation for the modified vector potential A which reads:

$$
\sigma \frac{\partial \mathrm{A}}{\partial t}-\nabla \cdot\left(\mu^{-1} \nabla \mathrm{A}\right)=\mathrm{J}_{s} .
$$

The electric and magnetic fields can be then recovered from A by suitably deriving this quantity.

From a mathematical point of view, both (2) and (3) are linear parabolic second order partial differential equations and they can numerically be implemented in the same way. The only difference is that equation (3) may be non-strictly parabolic due to the fact that the conductivity $\sigma$ vanishes in a part of the domain. Due to this fact, from the theoretical point of view (existence of solutions, numerical analysis of the discrete problem) equation (3) is more difficult to treat (see previous references and articles [8,17] in steady configurations). On the other hand, from a numerical point of view, many of the methods for the simulation of moving systems can be applied only to systems with a not too thin air-gap. For this reason, we have led numerical tests on equation (2) to show the flexibility of our method and its ability at reproducing the currents distribution in a fully conducting domain, composed of two parts, a free one rotating in sliding contact with the other. 


\subsection{Problem equations in the case of moving geometries}

From now on we study a two-dimensional problem defined in a domain $\Omega \subset \mathbb{R}^{2}$ which is assumed to be a Lipschitz polygonal bounded open set in $\mathbb{R}^{2}$. We refer to the equation (3) and we consider the case of moving geometries.

We denote by $\Omega_{1}$ a set containing the moving conducting part of the physical system, that is the rotor, chosen in such a way that $\Omega \backslash \Omega_{1}$ contains the still conducting part (i.e. $\Omega_{1}$ may contain a part of the air-gap but no part of the stator). Let $\Gamma:=\partial \Omega_{1}$, we assume that $\Gamma$ is a circle with center $O \in \Omega_{1}$ and that $\Omega_{1}$ is turning with the rotation speed $\omega \in C^{2}(] 0,+\infty[)$. We set $\Omega_{2}=\Omega \backslash \Omega_{1}$ and $\theta(t)=\int_{0}^{t} \omega(s) \mathrm{d} s$.

We call $r_{t}: \Omega_{1} \rightarrow \Omega_{1}$ the rotation operator at time $t$ which rotates the domain $\Omega_{1}$ of the angle $\theta=\theta(t)$ and $r_{-t}$ its inverse. We set $\Omega_{1}(t):=r_{t} \Omega_{1}(0)$ : of course the geometric set is always the same, but, the physical system may change since the physical quantities $\sigma, \varepsilon, \mu$ can be non-invariant with respect to $r_{t}$. Moreover, $r_{t}$ has always to be seen as a change in the system of coordinates which is naturally defined in $\Omega_{1}$. Accordingly we denote $\Omega=\bar{\Omega}_{1}(0) \cup \Omega_{2}$ and $\Omega(t)=\bar{\Omega}_{1}(t) \cup \Omega_{2}$.

In the case of moving bodies the equations (2) and (3) change accordingly to the movement law (see [19] for a short presentation) and we consider the formulation in term of Lagrangian variables.

We focus our attention on the equation (3). In the rest of the paper we rename the modified vector potential A as $u$ since the notation A can be misleading. We also adopt the notation $u=\left(u_{1}, u_{2}\right)$ where $u_{i}:=u_{\mid \Omega_{i}}$ for $i=1,2$ (the same for the parameters of the problem.)

The equation determining the dynamic of $u$ (as a function of Lagrangian variables) can be expressed in transmission form as follows:

$$
\begin{array}{lll}
\left(E_{1}\right) & \sigma_{1}(\mathbf{x}) \frac{\partial u_{1}}{\partial t}(\mathbf{x}, t)-\nabla \cdot\left(\mu_{1}^{-1}(\mathbf{x}) \nabla u_{1}\right)(\mathbf{x}, t)=0 & \left.\Omega_{1}(0) \times\right] 0, T[ \\
\left(E_{2}\right) & \sigma_{2}(\mathbf{x}) \frac{\partial u_{2}}{\partial t}(\mathbf{x}, t)-\nabla \cdot\left(\mu_{2}^{-1}(\mathbf{x}) \nabla u_{2}\right)=\mathrm{J}_{s}(\mathbf{x}, t) & \left.\Omega_{2} \times\right] 0, T[ \\
\left(I C_{1}\right) & u_{1}\left(r_{-t} \mathbf{x}, t\right)=u_{2}(\mathbf{x}, t) & \Gamma \times] 0, T[ \\
\left(I C_{2}\right) & \mu_{1}^{-1}\left(r_{-t} \mathbf{x}\right) \frac{\partial u_{1}}{\partial \mathbf{n}}\left(r_{-t} \mathbf{x}, t\right)=\mu_{2}^{-1}(\mathbf{x}) \frac{\partial u_{2}}{\partial \mathbf{n}}(\mathbf{x}, t) & \Gamma \times] 0, T[ \\
(B C) & u_{2}(\mathbf{x}, t)=0 & \partial \Omega \times] 0, T[ \\
(0 C) & u(\mathbf{x}, 0)=0 & \mathcal{C} \times\{0\}
\end{array}
$$

where the partial differential equations $\left(E_{1}\right)$ and $\left(E_{2}\right)$ are in the sense of distributions in $\Omega_{1}$ and $\Omega_{2}$ respectively and $\mathbf{n}$ is at every $\mathbf{x} \in \Gamma$ the unit vector normal outward to $\Omega_{2}$. We further assume that the data $J_{s} \in$ $L^{2}(] 0, T[\times \Omega)$ is a source current whose support is contained in $\Omega_{2}$. As regards to $(B C)$, for the sake of simplicity, we consider only homogeneous Dirichlet boundary conditions, but the general case (with non-homogeneous Dirichlet, Neumann and mixed Dirichlet-Neumann conditions) could be treated with the same tools. The interface conditions $\left(I C_{1}\right)$ and $\left(I C_{2}\right)$ are explained in what follows.

We define, for any $t \in \mathbb{R}$, the two rotation operators $R_{t}: H^{1}\left(\Omega_{1}\right) \rightarrow H^{1}\left(\Omega_{1}\right)$ and $\mathcal{R}_{t}: H^{1}\left(\Omega_{1}\right) \times H^{1}\left(\Omega_{2}\right) \rightarrow$ $H^{1}\left(\Omega_{1}\right) \times H^{1}\left(\Omega_{2}\right)$ as:

$$
R_{t}\left(u_{1}\right)(\mathbf{x})=u_{1}\left(r_{t} \mathbf{x}\right), \quad \mathcal{R}_{t}\left(u_{1}, u_{2}\right)(\mathbf{x}):=\left(R_{t}\left(u_{1}\right)(\mathbf{x}), u_{2}(\mathbf{x})\right)
$$

The function $\mathcal{R}_{-t}(u(t))$ turns out to be the physical magnetic potential, that is the magnetic potential expressed in Eulerian variables. The interface conditions $\left(I C_{1}\right),\left(I C_{2}\right)$ are the natural transmission conditions on this function: $\left(I C_{1}\right)$ stating the continuity of $\mathcal{R}_{-t}(u(t))$ (i.e. the continuity of $\left.\mathbf{E}\right)$ and $\left(I C_{2}\right)$ the equality of the flux (i.e. the continuity of the tangential component of $\mathbf{H})$. 
Remark 1.1. The same kind of formulation can be done in the context of the heat equation. If the unknown $u$ is the temperature in a moving structure, the behavior laws are exactly the same. That is, roughly speaking, the solid material point carries with itself its temperature when moving.

\subsection{Variational formulation}

We introduce the following notations:

$$
\begin{aligned}
& \mathcal{H}^{s}(\Omega)=H^{s}\left(\Omega_{1}\right) \times H^{s}\left(\Omega_{2}\right) \cap H_{0, \partial \Omega}^{1}\left(\Omega_{2}\right) \quad s \geq 1 \\
& \|u\|_{\star, s}^{2}=\left\|u_{1}\right\|_{s, \Omega_{1}}^{2}+\left\|u_{2}\right\|_{s, \Omega_{2}}^{2} \quad \forall u \in \mathcal{H}^{s}(\Omega) \quad \text { (broken norm); } \\
& |u|_{\star, s}^{2}=\left|u_{1}\right|_{s, \Omega_{1}}^{2}+\left|u_{2}\right|_{s, \Omega_{2}}^{2} \quad \forall u \in \mathcal{H}^{s}(\Omega) \quad \text { (broken semi-norm). }
\end{aligned}
$$

Moreover we simplify the notation by setting $\|\cdot\|_{\star}:=\|\cdot\|_{\star, 1}$. We know that $\mathcal{H}^{s}(\Omega)$ are Hilbert spaces with the natural norms and semi-norms defined in (6).

We now introduce the functional setting which is needed to write the variational formulation for the problem (4). Let

$$
\mathcal{U}^{t}:=\left\{u:=\left(u_{1}, u_{2}\right) \in \mathcal{H}^{1}(\Omega): u_{1}\left(r_{-t} \mathbf{x}\right)=u_{2}(\mathbf{x}) \quad \mathbf{x} \in \Gamma\right\}
$$

Remark that for every fixed $t, \mathcal{U}^{t}$ is a isomorphic to $H_{0}^{1}(\Omega)$ through the rotation operator $\mathcal{R}_{-t}$ and, as a consequence, it is a Hilbert space endowed with the norm $\|\cdot\|_{\star}$.

The natural space for trial functions is then:

$$
L^{2}\left(0, T ; \mathcal{U}^{t}\right):=\left\{u:=\left(u_{1}, u_{2}\right) \in L^{2}\left(0, T ; \mathcal{H}^{1}(\Omega)\right): u_{1}\left(r_{-t} \mathbf{x}\right)=u_{2}(\mathbf{x}) \quad \text { for a.a. } \mathbf{x} \in \Gamma, t \in\right] 0, T[\} .
$$

The space $L^{2}\left(0, T ; \mathcal{U}^{t}\right)$ is a closed subspace of $L^{2}\left(0, T ; \mathcal{H}^{1}(\Omega)\right)$ and it is isomorphic to $L^{2}\left(0, T ; H_{0}^{1}(\Omega)\right)$ through the rotation operator $\mathcal{R}_{-t}$.

Now, in order to obtain a variational equation in space, we need to introduce "spatial" test functions. The natural choice is:

$$
\mathcal{V}:=\left\{v \in L^{2}\left(0, T ; \mathcal{U}^{t}\right): \exists u \in H_{0}^{1}(\Omega) v \equiv \mathcal{R}_{t}(u) \text { for a.a. } \mathbf{x} \in \Omega, t \in\right] 0, T[\} .
$$

A function $v \in \mathcal{V}$ corresponds to a unique function $u \in H_{0}^{1}(\Omega)$ that we let evolve in time through the rotation $\mathcal{R}_{t}$. Then its behavior in time is completely fixed by the rotation. In our non standard setting, $\mathcal{V}$ is the counterpart of the space $H_{0}^{1}(\Omega)$ which is actually the standard space of test functions (in space) for heat type equations.

In this framework, by formal integration, we obtain the variational formulation for (4), denoted by Problem $(\mathrm{P})$ in what follows:

Find $u \in L^{2}\left(0, T ; \mathcal{U}^{t}\right) \cap C^{0}\left(0, T ; L^{2}(\mathcal{C})\right)$ such that for every $v \in \mathcal{V}$ :

$$
\left(\sigma \frac{\partial u}{\partial t}, v\right)_{\Omega}+\int_{\Omega_{1}} \mu^{-1} \nabla u_{1} \cdot \nabla v_{1} \mathrm{~d} \Omega+\int_{\Omega_{2}} \mu^{-1} \nabla u_{2} \cdot \nabla v_{2} \mathrm{~d} \Omega=\left(\mathrm{J}_{s}, v\right)_{\Omega_{2}}
$$

where $(\cdot, \cdot)_{D}$ denotes the $L^{2}(D)$ scalar product over a domain $D$.

In order to give a meaning to the equation (8), we need to be sure that it can be interpreted in the distributional sense in time. In particular, we simply need to prove that the quantity $\left(\sigma \frac{\partial u}{\partial t}, v\right)_{\Omega}$ is a distribution in time. We have, for any $\varphi \in \mathcal{D}(] 0, T[)$ :

$$
\left\langle\left(\sigma \frac{\partial u}{\partial t}, v\right)_{\Omega}, \varphi\right\rangle=-\int_{0}^{T}\left(\sigma u, \frac{\partial(v \varphi)}{\partial t}\right)_{\Omega}=-\int_{0}^{T}\left(\sigma u, v \frac{\partial \varphi}{\partial t}\right)_{\Omega}-(\sigma u, \varphi \mathbf{V} \cdot \nabla v)_{\Omega_{1}} \mathrm{~d} t
$$


where $\mathbf{V}$ is the rotation speed of $\Omega_{1}$, the duality pairing in the left hand side is the one between $\mathcal{D}(] 0, T[)$ and $\mathcal{D}^{\prime}(] 0, T[)$ and, in the right hand side, the integral is well defined and bounded by the norms of $u$ and $v$ associated to their own spaces and the norm of $\varphi$ in $C^{1}(] 0, T[)$.

Moreover, taking formally $v=u$ in (9) and by using the chain rule in the right hand side, we have:

$$
2\left\langle\left(\sigma \frac{\partial u}{\partial t}, u\right)_{\Omega}, \varphi\right\rangle=\left\langle\frac{\partial}{\partial t}(\sigma u, u)_{\mathcal{C}}, \varphi\right\rangle=-\int_{0}^{T}(\sigma u, u)_{\mathcal{C}} \frac{\partial \varphi}{\partial t} \mathrm{~d} t .
$$

As a consequence, if $u$ is solution of $(\mathrm{P})$, the following holds:

$$
\frac{1}{2} \frac{\partial}{\partial t}(\sigma u, u)_{\Omega}+\int_{\Omega_{1}} \mu^{-1} \nabla u_{1} \cdot \nabla u_{1} \mathrm{~d} \Omega+\int_{\Omega_{2}} \mu^{-1} \nabla u_{2} \cdot \nabla u_{2} \mathrm{~d} \Omega=\left(\mathrm{J}_{s}, u\right)_{\Omega_{2}} .
$$

In the following we will adopt the notation:

$$
a(u, v)=\int_{\Omega_{1}} \mu^{-1} \nabla u_{1} \cdot \nabla v_{1} \mathrm{~d} \Omega+\int_{\Omega_{2}} \mu^{-1} \nabla u_{2} \cdot \nabla v_{2} \mathrm{~d} \Omega .
$$

Remark 1.2. The problem $(\mathrm{P})$ is not standard due to two factors:

1) the $L^{2}\left(0, T ; \mathcal{U}^{t}\right)$ functional setting;

2) the conductivity $\sigma$ is not strictly positive, there is a coupling of the heat equation in the conductor with the Laplace one in the non-conducting part.

In the following we use the Faedo-Galerkin method to show that a solution exists and an a priori estimate to prove its uniqueness.

The first point has already been partially considered in the interpretation of problem (P) (see also Rem. 1.5) and the second will be taken into account in the next section in the construction of the Faedo-Galerkin approximation spaces.

\subsection{Well posedness of the continuous problem}

This section is devoted to the proof of the next theorem:

Theorem 1.3. Let $\mathrm{J}_{s} \in L^{2}\left(0, T ; L^{2}\left(\Omega_{2}\right)\right)$ be a given current source. Then, the problem $(P)$ is uniquely solvable. Moreover the solution $u$ of $(P)$ solves also (4) when $\left(E_{i}\right), i=1,2$ are intended in the sense of distributions.

Proof of Theorem 1.3. We start with the uniqueness which is obtained by an a priori stability estimate. Via the Poincaré inequality, recalling $(B C)$ in $(4)$, we have that:

$$
\left\|u_{2}\right\|_{0, \Omega_{2}} \leq C\left(\Omega_{2}\right)\left|u_{2}\right|_{1, \Omega_{2}} \text {. }
$$

Integrating in time (11) and by the Cauchy-Schwartz inequality, we obtain:

$$
\|\sqrt{\sigma} u\|_{0, \mathcal{C}}^{2}+\frac{1}{2} \int_{0}^{T}\left(\left|\sqrt{\mu} u_{1}\right|_{1, \Omega_{1}}^{2}+\left|\sqrt{\mu} u_{2}\right|_{1, \Omega_{2}}^{2}\right) \mathrm{d} t \leq C \int_{0}^{T}\left\|J_{s}\right\|_{0, \Omega_{2}}^{2} \mathrm{~d} t .
$$

This estimate gives directly the uniqueness of the solution by recalling that the traces are continuous at $\partial \mathcal{C}$. If $\mathrm{J}_{s} \equiv 0$ and $u_{\mid \partial \mathcal{C}}=0$ then $u=0$ almost everywhere in $\Omega$.

We now pass to the existence and for that we use the Faedo-Galerkin method (see [14], Vol. 5, for details). We solve the system (8) for a suitable sequence of nested finite dimensional spaces $\left\{\mathcal{U}_{N}^{t}\right\}_{N \geq 1}$ such that:

$$
\mathcal{U}_{N}^{t} \subset \mathcal{U}_{N+1}^{t} \subset \mathcal{U}^{t} \quad \forall N>0 \quad \text { and } \bigcup_{N \geq 1} \mathcal{U}_{N}^{t} \quad \text { dense in } \quad \mathcal{U}^{t}
$$

then by using the stability estimate (13), we prove the existence of the solution. 
The procedure is quite standard in the case of a completely conducting system while in the other case we have to pay attention to the fact that the equations we are dealing with are not strictly parabolic.

Thanks to the definition (7), in what follows we construct a suitable sequence of approximation spaces in $H_{0}^{1}(\Omega)$ and then, by applying the operator $\mathcal{R}_{t}$ we obtain $\left\{\mathcal{U}_{N}^{t}\right\}_{N}$.

We introduce the following $L^{2}$-orthogonal decomposition in $H_{0}^{1}(\Omega)$ :

$$
H_{0}^{1}(\Omega)=H_{0}^{1}(\mathcal{I}) \stackrel{\perp}{\oplus}\left(H_{0}^{1}(\mathcal{I})\right)^{\perp} ; \quad u=u_{I}+u_{C} \quad u \in H_{0}^{1}(\Omega), u_{I} \in H_{0}^{1}(\mathcal{I})
$$

the symbol $\stackrel{\perp}{\oplus}$ stands for the $L^{2}$-orthogonal direct sum, where, of course, the functions in $H_{0}^{1}(\mathcal{I})$ are extended by zero outside the insulator $\mathcal{I}$. Since the two spaces $H_{0}^{1}(\mathcal{I})$ and $\left(H_{0}^{1}(\mathcal{I})\right)^{\perp}$ are separable Hilbert spaces, it is always possible to find two sequences of nested finite dimensional spaces such that

$$
\begin{array}{ll}
V_{N} \subset V_{N+1} \subset H_{0}^{1}(\mathcal{I}), & \bigcup_{N \geq 1} V_{N} \quad \text { dense in } \quad H_{0}^{1}(\mathcal{I}) \\
W_{N} \subset W_{N+1} \subset\left(H_{0}^{1}(\mathcal{I})\right)^{\perp}, & \bigcup_{N \geq 1} W_{N} \quad \text { dense in } \quad\left(H_{0}^{1}(\mathcal{I})\right)^{\perp} .
\end{array}
$$

Finally we define $\mathcal{U}_{N}^{t}:=\mathcal{R}_{t} V_{N} \stackrel{\perp}{\oplus} \mathcal{R}_{t} W_{N}$. It is not hard to see that this space verifies (14). Note that the movement is exactly taken into account in the construction of these nested finite dimensional spaces.

We have now to study the existence of a solution for the variational problem (P) when replacing $\mathcal{U}^{t}$ with $\mathcal{U}_{N}^{t}$. After defining suitable basis for the spaces $V_{N}$ and $W_{N}$, for any $u_{N}, v_{N} \in \mathcal{U}_{N}^{t}$, the integral $\int_{\Omega} \sigma u_{N} v_{N} \mathrm{~d} \Omega$ is equal to $\left(M(t) v_{N}, u_{N}\right)$ where $M(t)$ is the usually called mass matrix and $u_{N}, v_{N}$ denote here the coefficients defining $u_{N}$ and $v_{N}$ in the chosen basis (we use the same notation since it is helpful and not misleading.)

Due to the fact that $\sigma=0$ at $\mathcal{I}$, we clearly have that the matrix $M(t)$ has the form (when a right ordering is chosen)

$$
M(t)=\left(\begin{array}{cc}
0 & 0 \\
0 & M_{W}(t)
\end{array}\right)
$$

where $M_{W}(t)$ is the mass matrix associated only to the space $\mathcal{R}_{t} W_{N}$.

It is easy to show that the matrix $M_{W}(t)$ is non-singular: for every given $w_{N} \in \mathcal{R}_{t} W_{N}$ such that $w_{N}^{T}(t) M_{W}(t) w_{N}(t)=0$ we want to show that $w_{N}=0$. We surely have $w_{N}=0$ over $\mathcal{C}$ and this means in particular that $w_{N \mid \partial \mathcal{C}}=0$. Since $w_{N \mid \partial \Omega}=0$ and $w_{N} \in \mathcal{R}_{t}\left(H_{0}^{1}(\mathcal{I})\right)^{\perp}$, we deduce that $w_{N}=0$ also over $\mathcal{I}$ and so that $w_{N}$ is identically 0 . The problem (8) on the finite dimensional spaces $\mathcal{U}_{N}^{t}$ reads:

Find $\quad u_{N} \in L^{2}\left(0, T ; \mathcal{U}_{N}^{t}\right) \cap C^{0}\left(0, T ; L^{2}(\mathcal{C})\right)$ such that, for every $v \in \mathcal{U}_{N}^{t}$ :

$$
\left(\sigma \frac{\partial u_{N}}{\partial t}, v_{N}\right)_{\mathcal{C}}+a\left(u_{N}, v_{N}\right)=\left(\mathrm{J}_{s}, v_{N}\right)_{\Omega_{2}}
$$

and it can be easily written in matrix form and using our decomposition. The system reads

$$
\frac{\mathrm{d}}{\mathrm{d} t}\left[\left(\begin{array}{cc}
0 & 0 \\
0 & M_{W}(t)
\end{array}\right)\left(\begin{array}{c}
u_{V}(t) \\
u_{W}(t)
\end{array}\right)\right]+\left(\begin{array}{cc}
A_{V V}(t) & A_{V W}(t) \\
A_{W V}(t) & A_{W W}(t)
\end{array}\right)\left(\begin{array}{c}
u_{V}(t) \\
u_{W}(t)
\end{array}\right)=\left(\begin{array}{c}
\mathrm{J}_{V}(t) \\
\mathrm{J}_{W}(t)
\end{array}\right) .
$$

We can obtain $u_{V}(t)$ as a function of $u_{W}(t)$ from the first set of equations since, thanks to our construction, the sub-matrix $A_{V V}(t)$ is surely non-singular at every time $t$ and $u_{W}(t)$ from the second one by solving a first order ordinary differential equation.

By using the a priori stability estimate (13) applied on the sequence $u_{N}$, there exists a subsequence of $u_{N}$ (called again $u_{N}$ for convenience), such that $u_{N} \rightarrow u$ in $L^{2}\left(0, T ; \mathcal{U}^{t}\right)$. Let now $v$ be any function in $\mathcal{U}^{t}$ and 
$v_{N} \in \mathcal{U}_{N}^{t}$ be a sequence strongly convergent to $v$ in $\mathcal{U}^{t}$. If we let $N$ go to infinity; we obtain (8). The second statement of the theorem is now straightforward.

In the next theorem we prove that, when the datum is regular, the solution $u$ of $(\mathrm{P})$ is in truth more regular and it belongs to $H^{1}\left(0, T ; L^{2}(\mathcal{C})\right) \cap L^{\infty}\left(0, T ; \mathcal{U}^{t}\right)$. Using this regularity, we immediately have that $u$ verifies $\left(E_{i}\right)$ in (4) in the sense of $L^{2}\left(\Omega_{i}\right), i=1,2$ and that the boundary and transmission conditions are naturally fitted.

Theorem 1.4. Let $\mathrm{J}_{s} \in H^{1}\left(0, T ; L^{2}(\Omega)\right)$ and $\mu$ be such that $\mu^{-1} \in W^{1, \infty}\left(\Omega_{1}\right)$. Then, the solution $u$ of problem $(P)$ belongs to $H^{1}\left(0, T ; L^{2}(\mathcal{C})\right) \cap L^{\infty}\left(0, T ; \mathcal{U}^{t}\right)$.

Proof of Theorem 1.4. We use the same Faedo-Galerkin approximation spaces (and also the same notation) as in the previous proof. The solution $u_{N}$ of (15) can be written as $u_{N}(x, t)=\sum_{i=1}^{N} u_{i}(t) \phi_{i}(x, t)$ where $\phi_{i}(x, t)$ are the basis functions for $\mathcal{U}_{N}^{t}$. We suppose without loss of generality that $\phi_{i}(x, t) \in \mathcal{H}^{2}(\Omega)$. Now, we choose the following test function in (15):

$$
v_{N}(x, t)=\sum_{i=1}^{N} \frac{\mathrm{d} u_{i}}{\mathrm{~d} t}(t) \phi_{i}(x, t)= \begin{cases}\frac{\partial u_{N}}{\partial t}-\mathbf{V} \cdot \nabla u_{N} & \text { on } \Omega_{1} \\ \frac{\partial u_{N}}{\partial t} & \text { on } \Omega_{2}\end{cases}
$$

where $\mathbf{V}$ is the rotation speed of $\Omega_{1}$. The function $v_{N}$ is admissible by construction and plugging it in (15) we get

$$
\int_{\mathcal{C}} \sigma\left|\frac{\partial u_{N}}{\partial t}\right|^{2}+a\left(u_{N}, \frac{\partial u_{N}}{\partial t}\right)=\int_{\Omega_{2}} \mathrm{~J}_{s}\left(\frac{\partial u_{N}}{\partial t}-\mathbf{V} \cdot \nabla u_{N}\right)+\int_{\Omega_{1}} \mu^{-1} \nabla u_{N} \cdot \nabla\left(\mathbf{V} \cdot \nabla u_{N}\right)+\int_{\mathcal{C}} \sigma \frac{\partial u_{N}}{\partial t} \mathbf{V} \cdot \nabla u_{N}
$$

We estimate the three terms in the right hand side starting from the last one:

$$
\int_{\mathcal{C}} \sigma \frac{\partial u_{N}}{\partial t} \mathbf{V} \cdot \nabla u_{N} \mathrm{~d} \Omega \leq \frac{1}{4} \int_{\Omega_{1}} \sigma\left|\frac{\partial u_{N}}{\partial t}\right|^{2} \mathrm{~d} \Omega+C\left|u_{N}\right|_{\star, \Omega}^{2}
$$

About the second term, using the chain rule, integrating by parts and reminding that $\mathbf{V} \cdot \mathbf{n}=0$, we have:

$$
\int_{\Omega_{1}} \mu^{-1} \nabla u_{N} \cdot \nabla\left(\mathbf{V} \cdot \nabla u_{N}\right)=\int_{\Omega_{1}} \mu^{-1} \nabla u_{N}(\nabla \mathbf{V} \cdot \nabla) u_{N}+\int_{\Omega_{1}} \operatorname{div}\left(\mu^{-1} \mathbf{V}\right)\left|\nabla u_{N}\right|^{2} \leq C(\mu, \omega)\left|u_{N}\right|_{\star, \Omega_{1}}^{2} .
$$

Finally, about the first one, we integrate it in time and we obtain the equality:

$$
\int_{0}^{t} \int_{\Omega} \mathrm{J}_{s}\left(\frac{\partial u_{N}}{\partial t}-\mathbf{V} \cdot \nabla u_{N}\right)=-\int_{\Omega_{2}} \int_{0}^{t} \frac{\partial \mathrm{J}_{s}}{\partial t} u_{N}+\int_{\Omega_{2}} \mathrm{~J}_{s}(\cdot, t) u_{N}(\cdot, t)-\int_{\Omega_{2}} \int_{0}^{t} \mathrm{~J}_{s} \mathbf{V} \cdot \nabla u_{N} .
$$

Then, we have that

$$
\text { the right hand side of }(19) \leq C|| \mathrm{J}_{s}\left\|_{H^{1}\left(0, T ; L^{2}(\Omega)\right)}\right\| u_{N}(\cdot, t) \|_{L^{\infty}\left(0, t, \mathcal{H}_{0}^{1}(\Omega)\right)} .
$$

By using these estimates in (16), and integrating in time, we obtain:

$$
\int_{\mathcal{C}} \sigma\left|\frac{\partial u_{N}}{\partial t}\right|^{2} \mathrm{~d} \Omega+\left\|u_{N}\right\|_{L^{\infty}\left(0, T ; \mathcal{U}^{t}\right)} \leq C\left\|\mathrm{~J}_{s}\right\|_{H^{1}\left(0, T ; L^{2}(\Omega)\right)}^{2} .
$$


There is then a subsequence of $u_{N}$ (that we call again $u_{N}$ for convenience) which converge weakly also in $H^{1}\left(0, T ; L^{2}(\mathcal{C})\right) \cap L^{\infty}\left(0, T ; \mathcal{U}^{t}\right)$. By uniqueness, the weak limit is again $u$ and this achieves the proof.

Remark 1.5. Thanks to this regularity results, we have that $\sigma \frac{\partial u}{\partial t} \in L^{2}\left(0, T ; L^{2}(\mathcal{C})\right)$ and, as a consequence, the term $\left(\sigma \frac{\partial u}{\partial t}, v\right)_{\Omega}$ belongs to $L^{2}(0, T)$. This means that equation (8) can now be intended in the sense of $L^{2}(0, T)$ and it is then suitable for the definition of the finite elements discretization.

\section{The SPATIAL DisCRETIZATION}

In the previous section we proved that our continuous problem is well posed. We want to propose a finite element algorithm, but if we think of solving this problem with a standard Galerkin method, the time dependent transmission condition $\left(I C_{1}\right)$ in $(4)$ has to be exactly verified. This fact imposes very heavy calculations since it is necessary to modify the mesh for the spatial discretization at every time step or to impose a heavy constraint between the time discretization step and the spatial one.

In the following we propose a non-conforming approximation of the problem (8); non-matching grids are used at every time step and the transmission condition is weakly imposed by means of a mortar element method technique (see [4]). Due to the time dependence of the coupling condition, namely $\left(I C_{1}\right)$ in (4), this technique leads us to a sliding mesh-mortar method which has been first introduced in the context of spectral approximation in [3].

Let $\mathcal{T}_{1, h_{1}}$ and $\mathcal{T}_{2, h_{2}}$ two families of curved triangulations for $\Omega_{1}$ and $\Omega_{2}$ respectively such that:

$$
\overline{\bigcup_{K \in \mathcal{T}_{1, h_{1}}} K} \equiv \overline{\Omega_{1}} \quad \overline{\bigcup_{K \in \mathcal{T}_{2, h_{2}}} K} \equiv \overline{\Omega_{2}} .
$$

We assume only the triangles with one edge on $\Gamma$ are curved and that $\mathcal{T}_{i, h_{i}}, i=1,2$ are regular in the sense of Ciarlet (see [13] for details), non-matching at the interface $\Gamma$. We denote by $h_{1}$ and $h_{2}$ their sizes and by $h$ the maximum between $h_{1}$ and $h_{2}$. For what concerns the analysis of the approximation properties for curved finite elements we refer e.g. to [6].

We denote by $\mathcal{T}_{i, h_{i}}^{\Gamma}, i=1,2$ the two discretizations of $\Gamma$ that are obtained by taking the traces of $\mathcal{T}_{1, h_{1}}$ and $\mathcal{T}_{2, h_{2}}$ on $\Gamma$. Let $h_{i}^{\Gamma}, i=1,2$, be the mesh sizes of these decompositions of $\Gamma$; we assume that there exist two constants $c, C \in \mathbb{R}$, such that

$$
c \leq \frac{h_{1}^{\Gamma}}{h_{2}^{\Gamma}} \leq C .
$$

With this assumption, we do not loose any generality since the interface $\Gamma$ is fictitious and can be set where it is more convenient: the physical system is homogeneous in a neighborhood of $\Gamma$. Then there is no interest in dealing with decompositions of very different sizes. Nevertheless, if one wants to use meshes that do not satisfy (21), then the results that follow still hold through a much more technical procedure.

Since the sub-domains have necessarily curved geometries, we need to introduce non-affine finite element spaces. According to [13], we denote by $\hat{K}$ the reference triangle and for every $K \in \mathcal{T}_{1, h_{1}} \cup \mathcal{T}_{2, h_{2}}$ we define the application $F_{K}: \hat{K} \rightarrow K$. Thanks to the regularity of $\Gamma$ it is easy to see that all these applications are regular and invertible.

We introduce the following spaces of finite elements for $i=1,2$ :

$$
X_{i, h_{i}}=\left\{v_{i, h_{i}} \in C^{0}\left(\bar{\Omega}_{i}\right) \text { such that } v_{i,\left.h_{i}\right|_{\partial \Omega}}=0 \quad \text { and } \quad v_{i,\left.h_{i}\right|_{K}} \circ F_{K} \in \mathcal{P}_{k}(\hat{K}) \quad \forall K \in \mathcal{T}_{i, h_{i}}\right\}
$$


In order to weakly impose the interface condition, we have to define a suitable space of Lagrange multipliers. For every $e \in \mathcal{T}_{2, h_{2}}^{\Gamma}$, we denote by $\left.f_{e}:\right] 0,1[\rightarrow e$ an application which associates the reference interval $] 0,1[$ to $e$. According to the mortar method theory, we choose the space of Lagrange multipliers as follows (the other choice turns out to be completely equivalent):

$$
M_{h}=\left\{\varphi_{h} \in C^{0}(\Gamma) \text { such that } \varphi_{\left.h\right|_{e}} \circ f_{e} \in \mathcal{P}_{k}(] 0,1[) \quad \forall e \in \mathcal{T}_{2, h_{2}}^{\Gamma}\right\} .
$$

In the mortar method terminology, this means that we choose the rotor as master and the stator as slave.

According to [7], we define the following approximation space:

$$
\begin{aligned}
\mathcal{U}_{h}^{t}=\left\{v_{h}=\left(v_{1, h}, v_{2, h}\right)\right. & \in X_{1, h} \times X_{2, h} \text { such that: } \\
& \left.\int_{\Gamma}\left(v_{1, h}\left(r_{-t} \mathbf{x}\right)-v_{2, h}(\mathbf{x})\right) \varphi_{h}(\mathbf{x}) \mathrm{d} \Gamma=0 \quad \forall \varphi_{h} \in M_{h}\right\}
\end{aligned}
$$

at every fixed time $t$. The constraint (25) is time dependent and it is the discrete weak version of the interface condition $\left(I C_{1}\right)$ in the continuous problem (4).

For the approximation properties of the family of discrete spaces $\mathcal{U}_{h}^{t}$ and for the coerciveness of the bilinear form $a(\cdot, \cdot)$ defined in $(12)$ with respect to the norm $\|\cdot\|_{\star}$, we refer to $[7]$ or $[4]$.

Remark 2.1. Note that our theory allows also for using different degrees of polynomials in the two domains, say $k_{1}$ and $k_{2}$. In this case the Lagrange multiplier space $M_{h}$ should be of continuous functions which are locally polynomials of degree $k_{2}$.

Remark 2.2. According to $[4,7]$ the definition of the Lagrange multiplier space is generally rather more complex than in (23). In our case the interface $\Gamma$ is a manifold without boundary, while, in the case of several sub-domains, the interfaces between adjacent sub-domains are compact manifolds with boundary (geometrically speaking): the space of Lagrange multipliers can not be chosen as one of the space of traces (as we did in (23)), but only as a suitable subspace of it. We refer to [4] for the presentation and analysis of the mortar element method in the general case.

\section{Fully Discrete approximation}

We propose to use an implicit Euler scheme for the time discretization in the interval $[0, T]$ with $T>0$. Let $\delta t$ be the time step and $N$ an integer such that $T=N \delta t$. Since the time discretization involves also a "discretization" in time of the space of test functions and of the rotation operators, we shorten our notation in the following way:

$$
t^{n}=n \delta t, \quad v^{n} \in \mathcal{U}^{t^{n}}=\mathcal{U}^{n}, \quad v_{h}^{n} \in \mathcal{U}_{h}^{t^{n}}=\mathcal{U}_{h}^{n}, \quad R_{t^{n}}=R_{n}, \quad \mathcal{R}_{t^{n}}=\mathcal{R}_{n} .
$$

By means of this notation, we denote by $u^{n}$ the solution of the continuous problem $(\mathrm{P})$ evaluated at $t=t^{n}$. By applying implicit Euler scheme in time and the nonconforming Galerkin method in space, we obtain the fully discrete problem:

$$
\begin{aligned}
& \forall n=0, . ., N-1, \quad \text { find } u_{h}^{n+1} \in \mathcal{U}_{h}^{n+1} \text { such that: } \\
& \qquad \int_{\mathcal{C}} \sigma \frac{u_{h}^{n+1}-u_{h}^{n}}{\delta t} v_{h}^{n+1} \mathrm{~d} \Omega+a\left(u_{h}^{n+1}, v_{h}^{n+1}\right)=\left(\mathrm{J}_{s}^{n+1}, v_{h}^{n+1}\right)_{\Omega_{2}} \quad \forall v_{h}^{n+1} \in \mathcal{U}_{h}^{n+1} .
\end{aligned}
$$


Remark 3.1. Note that we simply propose one of the possible time stepping schemes. E.g. the fully discrete equation with Crank-Nicolson reads, for any $\theta \in[0,1]$ :

$$
\begin{aligned}
& \forall n=0, . ., N-1, \text { find } u_{h}^{n+1} \in \mathcal{U}_{h}^{n+1} \text { such that: } \forall v_{h}^{n+1} \in \mathcal{U}_{h}^{n+1} \\
& \int_{\mathcal{C}} \sigma \frac{u_{h}^{n+1}-u_{h}^{n}}{\delta t} v_{h}^{n+1} \mathrm{~d} \Omega+a\left(\theta u_{h}^{n+1}+(1-\theta) u_{h}^{n}, v_{h}^{n+1}\right)=\left(\theta \mathrm{J}_{s}^{n+1}+(1-\theta) \mathrm{J}_{s}^{n}, v_{h}^{n+1}\right)_{\Omega_{2}} .
\end{aligned}
$$

In principle, any other time stepping scheme can be chosen thanks to the flexibility of the method regarding the movement. No constraint is imposed between the time step and mesh size and/or the velocity of the rotor; this means that also variable and/or adaptive time stepping can be used without changes in the spatial discretization and in the construction of the approximation space $\mathcal{U}_{h}^{n}$.

Although the convergence of the scheme is studied only in the easy case of implicit Euler time stepping, there is no constraint to the application of high order schemes in time (as proposed in [27]).

\subsection{Convergence analysis}

Since the stability estimate is very similar to the one of the heat equation, we look directly to the error estimate for the proposed scheme.

Let $\Pi_{h}^{t}: \mathcal{U}^{t} \rightarrow \mathcal{U}_{h}^{t}$ be a time dependent projection operator onto the discrete space $\mathcal{U}_{h}^{t}$ that will be made precise later on according to the properties we shall need on it. We denote by $\Pi_{h}^{n}: \mathcal{U}^{n} \rightarrow \mathcal{U}_{h}^{n}$ this operator evaluated at time $t=t^{n}$.

We define:

$$
w_{h}^{n}=\Pi_{h}^{n} u^{n} \quad \text { and } \quad \xi_{h}^{n}=u_{h}^{n}-w_{h}^{n}
$$

By combining (8) and (26), recalling (27), we get the following equality for every $v_{h}^{n+1} \in \mathcal{U}_{h}^{n+1}$ :

$$
\begin{aligned}
\int_{\mathcal{C}} \sigma \frac{\xi_{h}^{n+1}-\xi_{h}^{n}}{\delta t} v_{h}^{n+1} \mathrm{~d} \Omega+a\left(\xi_{h}^{n+1}, v_{h}^{n+1}\right)= & \int_{\mathcal{C}} \sigma\left(\frac{\partial u^{n+1}}{\partial t}-\frac{w_{h}^{n+1}-w_{h}^{n}}{\delta t}\right) v_{h}^{n+1} \mathrm{~d} \Omega \\
& +a\left(u^{n+1}-w_{h}^{n+1}, v_{h}^{n+1}\right)-\int_{\Gamma} \frac{\partial u^{n+1}}{\partial \mathbf{n}}\left[v_{h}^{n+1}\right] \mathrm{d} \Gamma
\end{aligned}
$$

where we denote by $\left[v_{h}^{n}\right]$ the jump of $\mathcal{R}_{-n} v_{h}^{n}$ across the interface $\Gamma$, that is $\left.\left[v_{h}^{n}\right]=\left(R_{-n} v_{1, h}^{n}\right)\right)_{\mid \Gamma}-v_{2, h \mid \Gamma}^{n}$, by $\partial u^{n+1} / \partial \mathbf{n}$ the normal derivative of $u^{n+1}$ in the direction normal to $\Gamma$ outward to $\Omega_{2}$ and by $\frac{\partial u^{n+1}}{\partial t}$ the time derivative of $u$ computed at $t=t^{n+1}$. In the right hand side of (28), the first two terms depend on the approximation properties of the projector $\Pi_{h}^{n}$ and the last one is the consistency error due to our non-conforming (mortar) approximation.

By choosing $v_{h}^{n+1}=\xi_{h}^{n+1}$, multiplying by $\delta t$ both sides of (28) and denoting by

$$
\begin{aligned}
& E_{\operatorname{appr}}(n)= \sup _{v_{h}^{n} \in_{h}^{n}} \frac{a\left(u^{n}-w_{h}^{n}, v_{h}^{n}\right)-\int_{\Gamma} \frac{\partial u}{\partial \mathbf{n}}\left[v_{h}^{n}\right] \mathrm{d} \Gamma}{\left\|v_{h}^{n}\right\|_{\star}} ; \\
& E_{\partial t}(n)=\sup _{v_{h}^{n} \in \mathcal{U}_{h}^{n}} \frac{\int_{\mathcal{C}} \sigma\left(\frac{\partial u^{n}}{\partial t}-\frac{w_{h}^{n}-w_{h}^{n-1}}{\delta t}\right) v_{h}^{n} \mathrm{~d} \Omega}{\left\|\sqrt{\sigma} v_{h}^{n}\right\|_{0, \mathcal{C}}} ;
\end{aligned}
$$


we get the following estimate:

$$
\left\|\sqrt{\sigma} \xi_{h}^{n+1}\right\|_{0, \mathcal{C}}^{2}+\delta t a\left(\xi_{h}^{n+1}, \xi_{h}^{n+1}\right) \leq \int_{\mathcal{C}} \sigma \xi_{h}^{n+1} \xi_{h}^{n} \mathrm{~d} \Omega+\delta t E_{\operatorname{appr}}(n+1)\left\|\xi_{h}^{n+1}\right\|_{\star}+\delta t E_{\partial t}(n+1)\left\|\sqrt{\sigma} \xi_{h}^{n+1}\right\|_{0, \mathcal{C}}
$$

Then, by the Cauchy-Schwartz inequality and the ellipticity of $a$, we have:

$$
\left(1-\frac{\delta t}{T}\right)\left\|\sqrt{\sigma} \xi_{h}^{n+1}\right\|_{0, \mathcal{C}}^{2}+\alpha \delta t\left\|\xi_{h}^{n+1}\right\|_{\star}^{2} \leq\left\|\sqrt{\sigma} \xi_{h}^{n}\right\|_{0, \mathcal{C}}^{2}+\delta t\left(\frac{1}{\alpha} E_{\mathrm{appr}}^{2}(n+1)+T E_{\partial t}^{2}(n+1)\right)
$$

where we have denoted by $\alpha=\alpha(\mu)$ the coerciveness constant of the bilinear form $a(\cdot, \cdot)$ with respect to the broken norm $\|\cdot\|_{\star}$. Now, using recursively (30) and by Gronwall's Lemma, we obtain:

$$
\left\|\sqrt{\sigma} \xi_{h}^{n}\right\|_{0, \mathcal{C}}^{2}+\alpha \sum_{i=1}^{n} \delta t\left\|\xi_{h}^{i}\right\|_{\star}^{2} \leq C(T, \sigma, \mu)\left\{\left\|\sqrt{\sigma} \xi_{h}^{0}\right\|_{0, \mathcal{C}}^{2}+\sum_{i=1}^{n} \delta t\left(\frac{1}{\alpha} E_{\mathrm{appr}}^{2}(i)+T E_{\partial t}^{2}(i)\right)\right\} \quad \forall n=1, . ., N
$$

where $T$ is the final time, the constant $C(T, \sigma, \mu)$ depends on $T$ and on the parameters of the problem, namely $\sigma$ and $\mu$, but not on the mesh size and time step.

This inequality is of key importance in the study the error estimate for our fully discrete formulation (26). The next theorem states the optimality of the proposed method.

Theorem 3.2. Let $u \in L^{2}\left(0, T ; \mathcal{U}^{t}\right) \cap C^{0}\left(0, T ; L^{2}(\mathcal{C})\right)$ be the solution of problem (8). Let $r \geq 2$, we assume:

$$
\begin{aligned}
& u \in L^{\infty}\left(0, T ; \mathcal{H}^{r}(\Omega)\right) ; \\
& \frac{\partial u}{\partial t} \in L^{2}\left(0, T ; \mathcal{H}^{r}(\Omega)\right) ; \\
& \frac{\partial^{2} u}{\partial t^{2}} \in L^{2}\left(0, T ; L^{2}(\Omega)\right) .
\end{aligned}
$$

Then, the following error estimate holds for $1 \leq s \leq \min \{r-1, k\}$ :

$$
\begin{aligned}
\left\|\sqrt{\sigma}\left(u^{n}-u_{h}^{n}\right)\right\|_{0, \mathcal{C}}^{2} & +\alpha \sum_{i=1}^{n} \delta t\left\|u^{i}-u_{h}^{i}\right\|_{\star}^{2} \leq C(T, \sigma, \mu)\left\{h^{2 s} \sum_{i=1}^{n} \delta t\left|u^{i}\right|_{\star, s+1}^{2}\right. \\
& \left.+h^{2(s+1)}\left(\left\|u^{n}\right\|_{s+1, \mathcal{C}}^{2}+\left\|u^{0}\right\|_{s+1, \mathcal{C}}^{2}\right)+(\delta t)^{2}\left\|\frac{\partial^{2} u}{\partial t^{2}}\right\|_{L^{2}\left(0, T ; L^{2}(\mathcal{C})\right)}^{2}+h^{2 s}\left\|\frac{\partial u}{\partial t}\right\|_{L^{2}\left(0, T ; \mathcal{H}^{s}(\Omega)\right)}^{2}\right\}
\end{aligned}
$$

for all $n=1, . ., N$ and where the constant $C(T, \sigma, \mu)$ depends on the parameters of the problem, but neither on $\delta t$ nor on $h$.

The proof of this theorem requires some preliminary results. We start stating and proving few lemmas and, at the end, we shall prove the theorem.

Lemma 3.3. Let $u \in L^{2}\left(0, T ; \mathcal{U}^{t}\right) \cap C^{0}\left(0, T ; L^{2}(\mathcal{C})\right)$. Assume moreover that $u$ verifies the regularity assumption of Theorem 3.2. There exists a family of projectors

$$
\left\{\Pi_{h}^{n}\right\}_{n=0, \ldots, N}, \quad \Pi_{h}^{n}: \mathcal{U}^{n} \cap \mathcal{H}^{2}(\Omega) \rightarrow \mathcal{U}_{h}^{n}
$$

such that $\forall n=0, . ., N$ and for $1 \leq s \leq \min \{r-1, k\}$ :

$$
h^{-1}|| u^{n}-\Pi_{h}^{n} u^{n}||_{0, \Omega}+\left\|u^{n}-\Pi_{h}^{n} u^{n}\right\|_{\star} \leq C_{1} h^{s}\left|u^{n}\right|_{\star, s+1} .
$$


Proof of Lemma 3.3. Let $\mathcal{P}_{2}: L^{2}(\Gamma) \rightarrow M_{h}$ be the $L^{2}$-orthogonal projection to $M_{h}$ and $\mathcal{I}_{h}: \mathcal{H}^{2}(\Omega) \rightarrow X_{1, h} \times X_{2, h}$ the interpolation operator. We define now a finite element lifting operator $\mathcal{L}_{h}: M_{h} \rightarrow X_{2, h}$ such that for every $\phi_{h} \in M_{h}, \mathcal{L}_{h}\left(\phi_{h}\right)$ is a finite element function which takes the value zero at every internal node in $\Omega_{2}$.

Then we choose the operator $\Pi_{h}^{n}$ in the following way:

$$
w_{h}^{n} \equiv \Pi_{h}^{n} u^{n}=\mathcal{I}_{h} u^{n}+\mathcal{L}_{h}\left(\mathcal{P}_{2}\left(R_{-n}\left(\mathcal{I}_{h} u_{1}^{n}\right)_{\mid \Gamma}-\mathcal{I}_{h} u_{2}^{n}\right)_{\mid \Gamma}\right) .
$$

It is easy to see that $\Pi_{h}^{n} u^{n} \in \mathcal{U}_{h}^{n}$ for every $n=0, \ldots, N$. Similar operators have been often used in the context of mortar method and a first construction was proposed in [7].

Using the triangle inequality:

$$
\left\|u^{n}-\Pi_{h}^{n} u^{n}\right\|_{\star}^{2} \leq 2\left\|u^{n}-\mathcal{I}_{h} u^{n}\right\|_{\star}^{2}+2\left\|\mathcal{L}_{h}\left(\mathcal{P}_{2}\left(R_{-n}\left(\mathcal{I}_{h} u_{1}^{n}\right)-\mathcal{I}_{h} u_{2}^{n}\right)\right)\right\|_{1, \Omega_{2}}^{2}
$$

and, since the first term in the right hand side can be estimated in a standard way, we focus our attention on the second one.

Using scaling arguments between any triangle $K \in \mathcal{T}_{2, h_{2}}$ and the reference triangle, the following local inequalities

$$
\begin{aligned}
\left\|\mathcal{L}_{h}\left(\phi_{h}\right)\right\|_{0, K}^{2} & \leq C h_{K}\left\|\phi_{h}\right\|_{0, \Gamma_{K}}^{2} \\
\left|\mathcal{L}_{h}\left(\phi_{h}\right)\right|_{1, K}^{2} & \leq C h_{K}^{-1}\left\|\phi_{h}\right\|_{0, \Gamma_{K}}^{2}
\end{aligned}
$$

hold for every triangle $K \in \mathcal{T}_{2, h_{2}}$ such that $\bar{K} \cap \Gamma=\Gamma_{K} \neq \emptyset$; here $h_{K}$ is the diameter of $K$ and $C$ is a constant independent of the mesh size.

By summing up for every $K \in \mathcal{T}_{2, h_{2}}$ and assuming that $\mathcal{T}_{2, h_{2}}^{\Gamma}$ is quasi-uniform (see [13] for the definition) we obtain:

$$
h_{2}^{-1}\left\|\mathcal{L}_{h}\left(\phi_{h}\right)\right\|_{0, \Omega_{2}}^{2}+h_{2}\left|\mathcal{L}_{h}\left(\phi_{h}\right)\right|_{1, \Omega_{2}}^{2} \leq C\left\|\phi_{h}\right\|_{0, \Gamma}^{2} .
$$

By using (36) and the stability of the $L^{2}$ - projector $\mathcal{P}_{2}$ in (35) we obtain:

$$
\left\|u^{n}-\Pi_{h}^{n} u^{n}\right\|_{\star}^{2} \leq 2\left\|u^{n}-\mathcal{I}_{h} u^{n}\right\|_{\star}^{2}+C h_{2}^{-1}\left\|R_{-n}\left(\mathcal{I}_{h} u_{1}^{n}\right)-\mathcal{I}_{h} u_{2}^{n}\right\|_{0, \Gamma}^{2} ;
$$

and, on the other hand:

$$
\left\|u^{n}-\Pi_{h}^{n} u^{n}\right\|_{0, \Gamma}^{2} \leq 2\left\|u^{n}-\mathcal{I}_{h} u^{n}\right\|_{0, \Gamma}^{2}+C h_{2}\left\|R_{-n}\left(\mathcal{I}_{h} u_{1}^{n}\right)-\mathcal{I}_{h} u_{2}^{n}\right\|_{0, \Gamma}^{2} .
$$

By the triangle inequality and recalling that $R_{-n}\left(u_{1}^{n}\right)_{\mid \Gamma}=u_{2 \mid \Gamma}^{n}$, we have:

$$
\left\|R_{-n}\left(\mathcal{I}_{h} u_{1}^{n}\right)-\mathcal{I}_{h} u_{2}^{n}\right\|_{0, \Gamma}^{2} \leq 2\left\|R_{-n}\left(\mathcal{I}_{h} u_{1}^{n}\right)-R_{-n}\left(u_{1}^{n}\right)\right\|_{0, \Gamma}^{2}+\left\|u_{2}^{n}-\mathcal{I}_{h} u_{2}^{n}\right\|_{0, \Gamma}^{2} .
$$

Finally, (33) is obtained by using $(39)$ in $(37,38)$ and standard estimates for the interpolation operator.

Lemma 3.4. Let $\psi$ be a function belonging to $H^{1}(\Gamma), \omega \in C^{0}([0, T])$ the angular speed and $R_{n}$ the associated rotation operator at time $t=t^{n}$. The following inequality holds:

$$
\left\|\left(R_{-n}-R_{-(n-1)}\right) \psi\right\|_{0, \Gamma} \leq C \delta t\|\psi\|_{1, \Gamma}
$$

where the constant $C$ is independent of the time step. 
Proof of Lemma 3.4. We set:

$$
\varphi(\rho, \theta)=\psi(\rho \cos \theta, \rho \sin \theta), \quad \theta \in(0,2 \pi), \rho=\bar{r}
$$

where $(\rho, \theta)$ is the polar system of coordinates naturally associated to $\Omega_{1}$ with respect to its rotation center $O$ and $\bar{r}$ is its radius. It is easy to see that

$$
\left\|\left(R_{-n}-R_{-(n-1)}\right) \varphi\right\|_{0, \Gamma}=\sqrt{\bar{r}}\left\|\varphi(\bar{r}, \theta)-\varphi\left(\bar{r}, \theta-\omega^{n} \delta t\right)\right\|_{L^{2}(0,2 \pi)}
$$

where $\min _{t \in\left[t^{n}, t^{n+1}\right]}|\omega(t)| \leq \omega^{n} \leq \max _{t \in\left[t^{n}, t^{n+1}\right]}|\omega(t)|$.

Now we estimate the right hand side of (41) as:

$$
\left\|\varphi(\bar{r}, \theta)-\varphi\left(\bar{r}, \theta-\omega^{n} \delta t\right)\right\|_{L^{2}(0,2 \pi)}^{2} \leq \omega^{n} \delta t \int_{0}^{2 \pi} \mathrm{d} \theta \int_{\theta-\omega^{n} \delta t}^{\theta}\left(\frac{\partial \varphi}{\partial \theta}(\bar{r}, \xi)\right)^{2} \mathrm{~d} \xi
$$

We denote now $\chi(\bar{r}, \theta)=\int_{0}^{\theta}\left(\frac{\partial \varphi}{\partial \theta}(\bar{r}, \xi)\right)^{2} \mathrm{~d} \xi$ and we have:

$$
\left\|\left(\varphi(\bar{r}, \theta)-\varphi\left(\bar{r}, \theta-\omega^{n} \delta t\right)\right)\right\|_{L^{2}(0,2 \pi)}^{2} \leq \omega^{n} \delta t \int_{0}^{2 \pi}\left(\chi(\bar{r}, \theta)-\chi\left(\bar{r}, \theta-\omega^{n} \delta t\right)\right) \mathrm{d} \theta \leq\left(\omega^{n} \delta t\right)^{2} \chi(\bar{r}, 2 \pi)
$$

since $\chi$ is an increasing function.

Actually, $\chi(\bar{r}, 2 \pi)=\left\|\frac{\partial \varphi}{\partial \theta}\right\|_{L^{2}(0,2 \pi)}^{2}$ and (40) follows from (42) by passing to Cartesian coordinates. In particular the constant $C$ in $(40)$ is $C=C\left(\|\omega\|_{\infty}, \bar{r}\right)$.

Lemma 3.5. Let $u \in L^{2}\left(0, T ; \mathcal{U}^{t}\right) \cap C^{0}\left(0, T ; L^{2}(\mathcal{C})\right)$. Assume moreover that $u$ verifies the regularity assumption of Theorem 3.2. The following estimate holds:

$$
E_{\operatorname{appr}}(n)+E_{\partial t}(n) \leq C_{1} h^{s}\left|u^{n}\right|_{\star, s+1}+C_{2}(\delta t)^{1 / 2}\left\|\frac{\partial^{2} u}{\partial t^{2}}\right\|_{L^{2}\left(t^{n-1}, t^{n} ; L^{2}(\mathcal{C})\right)}+C_{3} h^{s}(\delta t)^{-1 / 2}\left\|\frac{\partial u}{\partial t}\right\| \|_{L^{2}\left(t^{n-1}, t^{n} ; \mathcal{H}^{s}(\Omega)\right)}
$$

where $C_{i}=C_{i}(\mu, \sigma)$, for $i=1,2,3$ do not depend on $h$ or $\delta t$.

Proof of Lemma 3.5. To shorten our notation, we set $\sigma \equiv 1$ on $\mathcal{C}$ and $\mu \equiv 1$ on $\Omega$. We estimate first the term $E_{\text {appr }}(n)$ and then $E_{\partial t}(n)$.

For what concerns $E_{\operatorname{appr}}(n)$ we have to pay attention to the boundary term. Since, by construction, the jump $\left[v_{h}^{n}\right]$ is orthogonal to the Lagrange multiplier space $M_{h}$, we have:

$$
\int_{\Gamma} \frac{\partial u^{n}}{\partial \mathbf{n}}\left[v_{h}^{n}\right] \mathrm{d} \Gamma=\inf _{\varphi_{h} \in M_{h}} \int_{\Gamma}\left(\frac{\partial u^{n}}{\partial \mathbf{n}}-\varphi_{h}\right)\left[v_{h}^{n}\right] \mathrm{d} \Gamma \leq \inf _{\varphi_{h} \in M_{h}}\left\|\frac{\partial u^{n}}{\partial \mathbf{n}}-\varphi_{h}\right\|_{-1 / 2, \Gamma}\left\|\left[v_{h}^{n}\right]\right\|_{1 / 2, \Gamma} .
$$

By the Aubin-Nitsche argument [23] and standard trace theorems [1], we have that:

$$
\inf _{\varphi_{h} \in M_{h}}\left\|\frac{\partial u^{n}}{\partial \mathbf{n}}-\varphi_{h}\right\|_{-1 / 2, \Gamma} \leq C h^{s}\left|u^{n}\right|_{\star, s+1} \quad \text { and } \quad\left\|\left[v_{h}^{n}\right]\right\|_{1 / 2, \Gamma} \leq\left\|v_{h}^{n}\right\|_{\star} .
$$


Combining (33, 44-45) and the definition of $E_{\mathrm{appr}}(n)$ in (29), we have

$$
E_{\text {appr }}(n) \leq C h^{s}\left|u^{n}\right|_{\star s+1} .
$$

On the contrary, the estimate for $E_{\partial t}(n)$ is quite technical. We have that:

$$
\left\|\frac{\partial u^{n}}{\partial t}-\frac{\Pi_{h}^{n} u^{n}-\Pi_{h}^{n-1} u^{n-1}}{\delta t}\right\|_{0, \mathcal{C}} \leq\left\|\frac{\partial u^{n}}{\partial t}-\frac{u^{n}-u^{n-1}}{\delta t}\right\|_{0, \mathcal{C}}+\left\|\frac{u^{n}-u^{n-1}}{\delta t}-\frac{\Pi_{h}^{n} u^{n}-\Pi_{h}^{n-1} u^{n-1}}{\delta t}\right\|_{0, \mathcal{C}}
$$

where the estimate of the first term in the right hand side is standard (see e.g. [27]), while the second term has still to be studied. By the triangle inequality, we have:

$$
\begin{aligned}
\left\|u^{n}-u^{n-1}-\left(\Pi_{h}^{n} u^{n}-\Pi_{h}^{n-1} u^{n-1}\right)\right\|_{0, \mathcal{C}} \leq & \left\|u^{n}-u^{n-1}-\mathcal{I}_{h}\left(u^{n}-u^{n-1}\right)\right\|_{0, \mathcal{C}} \\
& +\left\|\mathcal{L}_{h}\left(\mathcal{P}_{2}\left(R_{-n}\left(\mathcal{I}_{h} u_{1}^{n}\right)-\mathcal{I}_{h} u_{2}^{n}-R_{-(n-1)}\left(\mathcal{I}_{h} u_{1}^{n-1}\right)+\mathcal{I}_{h} u_{2}^{n-1}\right)\right)\right\|_{0, \mathcal{C}} ;
\end{aligned}
$$

where again it is the second term in the right hand side that we have to analyze. In what follows we set

$$
\mathcal{E}_{h}:=\left\|\mathcal{L}_{h}\left(\mathcal{P}_{2}\left(R_{-n}\left(\mathcal{I}_{h} u_{1}^{n}\right)-\mathcal{I}_{h} u_{2}^{n}-R_{-(n-1)}\left(\mathcal{I}_{h} u_{1}^{n-1}\right)+\mathcal{I}_{h} u_{2}^{n-1}\right)\right)\right\|_{0, \mathcal{C}}
$$

By means of (36), the stability of $L^{2}-$ orthogonal projection $\mathcal{P}_{2}$ and triangle inequality, we obtain:

$$
\mathcal{E}_{h} \leq \sqrt{h_{2}}\left\|R_{-n}\left(\mathcal{I}_{h} u_{1}^{n}\right)-R_{-(n-1)}\left(\mathcal{I}_{h} u_{1}^{n-1}\right)-\left(\mathcal{I}_{h} u_{2}^{n}-\mathcal{I}_{h} u_{2}^{n-1}\right)\right\|_{0, \Gamma} .
$$

By summing and subtracting $\left(u_{2}^{n}-u_{2}^{n-1}\right)_{\mid \Gamma}=\left(R_{-n}\left(u_{1}^{n}\right)-R_{-(n-1)}\left(u_{1}^{n-1}\right)\right)_{\mid \Gamma}$ and by the triangle inequality:

$$
\mathcal{E}_{h} \leq \sqrt{h_{2}}\left(\left\|R_{-n}\left(\mathcal{I}_{h} u_{1}^{n}-u_{1}^{n}\right)-R_{-(n-1)}\left(\mathcal{I}_{h} u_{1}^{n-1}-u_{1}^{n-1}\right)\right\|_{0, \Gamma}+\left\|\mathcal{I}_{h}\left(u_{2}^{n}-u_{2}^{n-1}\right)-\left(u_{2}^{n}-u_{2}^{n-1}\right)\right\|_{0, \Gamma}\right) .
$$

Now the first term in the right hand side of (48) can be bounded in the following way:

$$
\begin{aligned}
\left\|R_{-n}\left(\mathcal{I}_{h} u_{1}^{n}-u_{1}^{n}\right)-R_{-(n-1)}\left(\mathcal{I}_{h} u_{1}^{n-1}-u_{1}^{n-1}\right)\right\|_{0, \Gamma} \leq & \left\|R_{-n}\left(\mathcal{I}_{h}\left(u_{1}^{n}-u_{1}^{n-1}\right)-\left(u_{1}^{n}-u_{1}^{n-1}\right)\right)\right\|_{0, \Gamma} \\
& +\left\|\left[R_{-n}-R_{-(n-1)}\right]\left(\mathcal{I}_{h} u_{1}^{n-1}-u_{1}^{n-1}\right)\right\|_{0, \Gamma} .
\end{aligned}
$$

Then, coming back to the estimate of $\mathcal{E}_{h}$, by standard arguments we get:

$$
\mathcal{E}_{h} \leq C h^{s}\left|u^{n}-u^{n-1}\right|_{\star, s}+C \sqrt{h_{2}}\left\|\left(R_{-n}-R_{-(n-1)}\right)\left(\mathcal{I}_{h} u_{1}^{n-1}-u_{1}^{n-1}\right)\right\|_{0, \Gamma} .
$$

The estimate of the second term in the right hand side of (49) is then obtained by applying Lemma 3.4 to the function $\mathcal{I}_{h} u_{1}^{n-1}-u_{1}^{n-1}$. Finally we have:

$$
\mathcal{E}_{h} \leq h^{s}\left|u^{n}-u^{n-1}\right|_{\star, s}+\delta t h_{2}^{s}\left|u_{1}^{n}\right|_{s+1 / 2, \Gamma}
$$

Coming back now to the estimate of $E_{\partial t}(n)$, by using $(46,47)$ and $(50)$ we have then

$$
E_{\partial t}(n) \leq\left\|\frac{\partial u^{n}}{\partial t}-\frac{u^{n}-u^{n-1}}{\delta t}\right\|_{0, \mathcal{C}}+h^{s}\left\|\frac{u^{n}-u^{n-1}}{\delta t}\right\|_{\star, s}+h^{s}\left|u_{1}^{n}\right|_{s+1 / 2, \Gamma}
$$


By means of standard estimates for first order schemes in time (see e.g. [27]) and by the continuity of the trace operator $\gamma_{0}: H^{s}(\Omega) \rightarrow H^{s-1 / 2}(\Gamma)$ for $1 \leq s<k$, we get:

$$
E_{\partial t}(n) \leq C \sqrt{\delta t}\left\|\frac{\partial^{2} u}{\partial t^{2}}\right\|_{L^{2}\left(t^{n-1}, t^{n}, L^{2}(\mathcal{C})\right)}+\frac{h^{s}}{\sqrt{\delta t}}\left\|\frac{\partial u}{\partial t}\right\|_{L^{2}\left(t^{n-1}, t^{n}, \mathcal{H}^{s}(\Omega)\right)}+h^{s}\left|u^{n}\right|_{\star, s+1} .
$$

We are finally in the position to prove Theorem 3.2.

Proof of Theorem 3.2. Using the triangle inequality in (31) we easily obtain the following inequality:

$$
\begin{aligned}
\left\|u^{n}-u_{h}^{n}\right\|_{0, \mathcal{C}}^{2}+\alpha \sum_{i=1}^{n} \delta t\left\|u^{i}-u_{h}^{i}\right\|_{\star}^{2} \leq C(T, \mu, \sigma) & \left\{\left\|u^{n}-w_{h}^{n}\right\|_{0, \mathcal{C}}^{2}+\alpha \sum_{i=1}^{n} \delta t\left\|u^{i}-w_{h}^{i}\right\|_{\star}^{2}\right. \\
& \left.+\left\|u(0)-w_{h}^{0}\right\|_{0, \mathcal{C}}^{2}+\sum_{i=1}^{n} \delta t\left(\frac{1}{\alpha} E_{\text {appr }}^{2}(i)+T E_{\partial t}^{2}(i)\right)\right\} .
\end{aligned}
$$

In order to obtain the estimate (32) it is sufficient to apply the results of Lemmas 3.3 and 3.5 in the right hand side of (51).

\section{EFFECTS OF USING ISO-PARAMETRIC FINITE ELEMENTS}

In Section 2, we introduced curved finite element triangulations in both domains $\Omega_{1}$ and $\Omega_{2}$ and the advantage of using non-matching grids has been pointed out.

The use of curved finite elements is not suitable for a fast and easy implementation and makes the exact computation of the stiffness and mass matrix almost impossible. The remedy is to use, at the place of curved finite elements, iso-parametric finite elements for which the basis functions are polynomials and which allow for an exact numerical integration using Gaussian points.

The purpose of this section is the analysis of the non-matching method in order to take this additional factor into account. The fact of using such finite elements changes the definition of the approximation space (24-25). The constraint condition (25) no longer makes sense since, in general, neither $v_{1, h}$ nor $v_{2, h}$ in (25) are defined over $\Gamma$.

Due to the existing literature on the subject for a fixed domain, the only new point is the effect of using iso-parametric elements along the interface. We assume that it is the only place where a problem may raise by making the hypothesis that $\Omega$ has a polygonal boundary.

Now, it is easy to see from the analysis of the error that we carried out in Section 3 that we can focus our attention on deriving optimal approximation and consistency error bounds for the following static problem:

$$
\begin{aligned}
& \text { Find } \quad u \in H_{0}^{1}(\Omega) \text { such that: } \\
& a(u, v)=(f, v)_{\Omega} \quad \forall v \in H_{0}^{1}(\Omega)
\end{aligned}
$$

where the bilinear form $a$ is defined in (12), $\Omega=\Omega_{1}(0) \cup \Omega_{2}$ as in Section 1 and where the discretization relies on a space $\mathcal{U}_{h}^{0}$ (see (24-25)) that now takes into account the effect of using iso-parametric finite element approximations in both $\Omega_{1}$ and $\Omega_{2}$.

More precisely, according to [13], we introduce two families of iso-parametric finite elements $\left(\tilde{\mathcal{T}}_{1, h}, \tilde{X}_{1, h}\right)$ and $\left(\tilde{\mathcal{T}}_{2, h}, \tilde{X}_{2, h}\right)$ for $\Omega_{1}$ and $\Omega_{2}: \tilde{\mathcal{T}}_{1, h}$ (respectively $\left.\tilde{\mathcal{T}}_{2, h}\right)$ is an iso-parametric triangulation of $\Omega_{1}$ (resp. $\Omega_{2}$ ) of degree $k$ and $\tilde{X}_{1, h}$ (resp. $\tilde{X}_{2, h}$ ) is a finite element space of degree $k$ defined on $\tilde{\mathcal{T}}_{1, h}$ (resp. $\tilde{\mathcal{T}}_{2, h}$ ). For the sake of simplicity we shall suppose that both $\tilde{X}_{1, h}$ and $\tilde{X}_{2, h}$ are of degree $k$, but the same theory can be developed in the case of 
approximations of different degrees without any substantial change. Moreover we adopt the following notation:

$$
\begin{aligned}
\Omega_{1, h} & =\frac{\circ}{\bigcup_{K \in \tilde{\mathcal{T}}_{1, h}} K} ; \quad \Gamma_{1}^{h}=\partial \Omega_{1, h} ; \\
\Omega_{2, h} & =\frac{\circ}{\bigcup_{K \in \tilde{\mathcal{T}}_{2, h}} K} ; \quad \Gamma_{2}^{h}=\partial \Omega_{2, h} \backslash \partial \Omega ; \\
\Omega_{h} & =\Omega_{1, h} \cup \Omega_{2, h} .
\end{aligned}
$$

Finally we denote by $\tilde{\mathcal{T}}_{i, h}^{\Gamma}$ the trace of $\tilde{\mathcal{T}}_{i, h}$ over $\Gamma_{i}^{h}$, for $i=1,2$. Since $\Gamma_{1}^{h}$ differs from $\Gamma_{2}^{h}$, the condition (24) can not be imposed; we thus need to introduce some transfer operators. Here is an example that is natural, at least from an analytical point of view: recalling that $O$ is the rotation center of $\Omega_{1}$, we denote by $S_{1}: \Gamma \rightarrow \Gamma_{1}^{h}$, $S_{2}: \Gamma \rightarrow \Gamma_{2}^{h}, \Gamma \ni(\bar{r}, \theta) \mapsto\left(r_{i}(\theta), \theta\right) \in \Gamma_{i}^{h}$, the two one-to-one radial shift operators with respect to $O$. It is very easy to see that $S_{i} \in W^{1, \infty}\left(\mathbb{R}^{2}\right)^{2}$ and that the following property holds:

$$
\left|J S_{i}\right|=1+O\left(h^{k}\right) \quad i=1,2 ;
$$

where $J S_{i}$ denotes the Jacobian of $S_{i}$.

Accordingly we define the shift operators over functions, $\mathcal{S}_{i}: L^{2}\left(\Gamma_{i}^{h}\right) \rightarrow L^{2}(\Gamma)$ for $i=1,2$, such that:

$$
\mathcal{S}_{i}\left(u_{i}\right)(\mathbf{x})=u_{i}\left(S_{i} \mathbf{x}\right) \text { a.e. } \mathbf{x} \in \Gamma, \quad u_{i} \in L^{2}\left(\Gamma_{i}^{h}\right) .
$$

It is easy to see that these operators are $H^{s}$-continuous for $s \in[0,1]$ (with the standard convention that $H^{0}=L^{2}$ ) with continuity constants bounded independently of $h$.

By abuse of notation, when it is useful, we write $\mathcal{S}_{2}(v)$ instead of $\mathcal{S}_{2}\left(v_{\mid \Gamma_{2}^{h}}\right)$ for every $v \in H^{1}\left(\Omega_{2, h}\right)$ and, moreover, we denote by $\mathcal{S}_{2}\left(\tilde{X}_{2, h}\right)$ the range of $\mathcal{S}_{2}$ restricted to the set of traces of functions in $\tilde{X}_{2, h}$ (of course the same notation is also used for $\left.\mathcal{S}_{1}\right)$.

We are now in the position of defining a new approximation space for the solution of problem (52). Let $e \in \tilde{\mathcal{T}}_{2, h}^{\Gamma}$ be an edge on $\Gamma_{2}^{h}$, we denote by $\left.f_{e}:\right] 0,1[\rightarrow e$ a bi-continuous application between the reference element $] 0,1[$ and $e$. Following the same steps as in Section 2, we start from the space of Lagrange multipliers defined as:

$$
\tilde{M}_{h}=\left\{\varphi_{h} \in C^{0}\left(\Gamma_{2}^{h}\right) \text { such that } \varphi_{h \mid e} \circ f_{e} \in \mathcal{P}_{k}(] 0,1[) \quad \forall e \in \Gamma_{2}^{h}\right\} .
$$

We choose again the rotor as master but the other choice would be completely equivalent.

We define the following approximation space:

$$
\begin{aligned}
\tilde{X}_{h}=\left\{v_{h}=\left(v_{1, h}, v_{2, h}\right) \in \tilde{X}_{1, h} \times \tilde{X}_{2, h}\right. \text { such that: } & \\
& \left.\int_{\Gamma_{1}^{h}}\left(\mathcal{S}_{1} v_{1, h}(\mathbf{x})-\mathcal{S}_{2} v_{2, h}(\mathbf{x})\right) \mathcal{S}_{2} \varphi_{h}(\mathbf{x}) \mathrm{d} \Gamma=0 \quad \forall \varphi \in \tilde{M}_{h}\right\} .
\end{aligned}
$$

Remember that we focus our analysis on the spatial discretization thus, with respect to (25), there is no rotation operator $r_{-t}$ involved.

Let us define $\tilde{X}_{h}^{0}=\left\{v_{h} \in \tilde{X}_{h}\right.$ such that $\left.v_{h \mid \partial \Omega_{2, h} \backslash \Gamma_{2}^{h}}=0\right\}$.

According to the standard domain decomposition method, we propose to solve the following discrete problem:

$$
\text { Find } u_{h} \in \tilde{X}_{h}^{0} \text { such that } a_{h}\left(u_{h}, v_{h}\right)=\left(f, v_{h}\right)_{h} \quad \forall v_{h} \in \tilde{X}_{h}^{0}
$$


where

$$
\begin{aligned}
a_{h}\left(u_{h}, v_{h}\right) & =\int_{\Omega_{1, h}} \nabla u_{1, h} \nabla v_{1, h} \mathrm{~d} \Omega+\int_{\Omega_{2, h}} \nabla u_{2, h} \nabla v_{2, h} \mathrm{~d} \Omega ; \\
\left(f, v_{h}\right)_{h} & =\int_{\Omega_{1, h}} f v_{1, h} \mathrm{~d} \Omega+\int_{\Omega_{2, h}} f v_{2, h} \mathrm{~d} \Omega .
\end{aligned}
$$

Furthermore we define a suitable mesh-dependent broken norm as:

$$
\|u\|_{\star, h}^{2}:=\|u\|_{1, \Omega_{1, h}}^{2}+\|u\|_{1, \Omega_{2, h}}^{2}, \quad u_{\mid \Omega_{1, h}} \in H^{1}\left(\Omega_{1, h}\right), u_{\mid \Omega_{2, h}} \in H^{1}\left(\Omega_{2, h}\right) .
$$

Lemma 4.1. The bilinear form $a_{h}$ defined in (58) is uniformly coercive in $\tilde{X}_{h}$ equipped with the broken norm $\|\cdot\|_{\star, h} ;$ that is

$$
\exists \alpha>0 \text { such that } a_{h}\left(v_{h}, v_{h}\right) \geq \alpha\left\|v_{h}\right\|_{\star, h}^{2} \quad \forall v_{h} \in \tilde{X}_{h}
$$

where the constant $\alpha$ does not depend on the mesh size $h$.

The proof of this lemma is analogous to the standard one in the mortar element method context and it can be found in [7] for example.

Also the following lemma does not need a proof since it is the well known Second Strang lemma (also known as Berger-Scott-Strang lemma [23]):

Lemma 4.2 (Second strang). Let $u$ and $u_{h}$ be the solutions of problems (52) and (57) respectively. There exist two positive constants $C, C^{\prime}$ such that the following error estimate holds:

$$
\left\|u-u_{h}\right\|_{\star, h} \leq C \inf _{v_{h} \in \tilde{X}_{h}}\left\|u-v_{h}\right\|_{\star, h}+C^{\prime} \sup _{w_{h} \in \tilde{X}_{h}} \frac{\left(f, w_{h}\right)_{h}-a_{h}\left(u, w_{h}\right)}{\left\|w_{h}\right\|_{\star, h}} .
$$

In particular the first term in the right hand side of inequality (60) is the best approximation error while the second one is the consistency error: in the following we study separately these two contributions.

\subsection{Best approximation error}

We only have to follow the basic steps of the proof for the best approximation error in the case of the standard mortar element method which has been proposed in [7].

Lemma 4.3. Let $\Pi: H^{1}\left(\Omega_{1}\right) \rightarrow \mathcal{S}_{2}\left(\tilde{X}_{2, h}\right) \subset H^{1}(\Gamma)$ be the $L^{2}$-projector defined in the following way:

$$
\int_{\Gamma}\left(u_{\mid \Gamma}-\Pi u\right) \mathcal{S}_{2}\left(\phi_{h}\right) \mathrm{d} \Gamma=0 \quad \forall \phi_{h} \in \tilde{M}_{h}
$$

the following approximation property holds:

$$
\left\|u_{\mid \Gamma}-\Pi u\right\|_{0, \Gamma} \leq C h^{s}\|u\|_{s+1 / 2, \Omega} \quad \forall u \in H^{s+1 / 2}(\Omega), \quad s \in[1, k+1 / 2] .
$$

Proof of Lemma 4.3. We denote by $I_{h}^{2}$ the interpolation operator in $\Omega_{2, h}$, then, since $\Pi$ is an $L^{2}$-projection, we surely have that

$$
\left\|u_{\mid \Gamma}-\Pi u\right\|_{0, \Gamma} \leq\left\|u_{\mid \Gamma}-\mathcal{S}_{2}\left(I_{h}^{2} u\right)\right\|_{0, \Gamma}
$$


and, by using the triangle inequality and the $L^{2}$ stability of $\mathcal{S}_{2}$, we have:

$$
\left\|u_{\mid \Gamma}-\Pi u\right\|_{0, \Gamma} \leq\left\|u_{\mid \Gamma}-\mathcal{S}_{2} u_{\mid \Gamma_{2}^{h}}\right\|_{0, \Gamma}+C\left\|u_{\mid \Gamma_{2}^{h}}-\left(I_{h}^{2} u\right)_{\mid \Gamma_{2}^{h}}\right\|_{0, \Gamma_{2}^{h}} .
$$

The estimate of the second term in the right hand side is standard; we focus our attention on the first one and of course we want this term to be bounded by the right hand side of (62).

Let $(\rho, \theta)$ be the system of polar coordinates with respect to the rotation center $O$ of $\Omega_{1}$ and $\bar{r}$ be the radius of $\Omega_{1}$. Without loosing generality, we suppose that $B(3 \bar{r} / 2, O) \subset \Omega$ where $B(3 \bar{r} / 2, O)$ denotes the ball of center $O$ and radius $3 \bar{r} / 2$. For $h$ small enough, there exist $c \in L^{\infty}([0,2 \pi))$ such that $-\bar{r} / 2 \leq c(\theta) \leq \bar{r} / 2$ and the following equality holds:

$$
\| u_{\mid \Gamma}-\left.\mathcal{S}_{2} u_{\mid \Gamma_{2}^{h}}\right|_{0, \Gamma} ^{2}=\int_{0}^{2 \pi}\left(\int_{\bar{r}}^{\bar{r}+h^{k+1} c(\theta)} \frac{\partial u}{\partial r}(s, \theta) \mathrm{d} s\right)^{2} \mathrm{~d} \theta .
$$

By using the Sobolev embedding theorems we deduce that:

$$
\left\|u_{\mid \Gamma}-\mathcal{S}_{2} u_{\mid \Gamma_{2}^{h}}\right\|_{0, \Gamma}^{2} \leq C h^{2(k+1)}\left\|\frac{\partial u}{\partial r}\right\|_{L^{\infty}\left(\Omega_{1}\right)}^{2} \leq C h^{2(k+1)}\|u\|_{3, \Omega}^{2} .
$$

While, using Cauchy-Schwartz inequality, we have from (64):

$$
\left\|u_{\mid \Gamma}-\mathcal{S}_{2} u_{\mid \Gamma_{2}^{h}}\right\|_{0, \Gamma}^{2} \leq C h^{k+1} \int_{0}^{2 \pi} \int_{\bar{r}}^{\bar{r}+h^{k+1}|c(\theta)|}\left|\frac{\partial u}{\partial r}(s, \theta)\right|^{2} \mathrm{~d} s \mathrm{~d} \theta \leq C h^{k+1}|u|_{1, \Omega}^{2} .
$$

By standard interpolation theory, from (65) and (66), we surely obtain that

$$
\left\|u_{\mid \Gamma}-\mathcal{S}_{2} u_{\mid \Gamma_{2}^{h}}\right\|_{0, \Gamma} \leq C h^{s}\|u\|_{s+1 / 2, \Omega} \quad \forall s \in[1, k+1 / 2] .
$$

Using now (63), (67) and standard results on the interpolation operator, we have that (62) holds.

We want now to construct a function $v_{h} \in \tilde{X}_{h}$ which provides an optimal approximation error and we do that by means of the projector $\Pi$ defined and analyzed in the previous lemma.

Proposition 4.4. Let $I_{1}^{h}$ (resp. $I_{2}^{h}$ ) be the interpolant operator in $\Omega_{1, h}$ (resp. $\Omega_{2, h}$ ), $I_{h}=\left(I_{1}^{h}, I_{2}^{h}\right)$ and $\mathcal{L}_{h}$ : $\tilde{M}_{h} \rightarrow \tilde{X}_{2, h}$ an extension operator such that for every $\phi_{h} \in \tilde{M}_{h}, \mathcal{L}_{h}\left(\phi_{h}\right)$ takes the value zero at all the internal nodes in $\Omega_{2}$. Moreover, let $u \in H_{0}^{1}(\Omega)$ be the solution of the problem (52); we assume that $u \in H^{m}(\Omega)$ for some $m>1$. The function $v_{h}=\left(v_{1, h}, v_{2, h}\right) \in \tilde{X}_{h}$ defined as:

$$
v_{1, h}=I_{1}^{h} u \quad \text { and } \quad v_{2, h}=I_{2}^{h} u+\mathcal{L}_{h}\left(\mathcal{S}_{2}^{-1}\left(\Pi \mathcal{S}_{1}\left(I_{1}^{h} u\right)\right)-I_{2}^{h} u\right)
$$

is such that $v_{h} \in \tilde{X}_{h}$ and it verifies for every $1 \leq s \leq \min \{k, m-1\}$ :

$$
\begin{aligned}
& \| u-\left.v_{h}\right|_{\star, h} \leq C_{1} h^{s}|u|_{s+1, \Omega} ; \\
& \| u-\left.v_{h}\right|_{0, \Omega_{h}} \leq C_{2} h^{s+1}|u|_{s+1, \Omega} .
\end{aligned}
$$

Proof of Proposition 4.4. By means of (36) we have that

$$
\left\|u-v_{h}\right\|_{\star, h} \leq\left\|u-I_{h} u\right\|_{\star, h}+h^{-1 / 2}\left\|\mathcal{S}_{2}^{-1}\left(\Pi \mathcal{S}_{1}\left(I_{1}^{h} u\right)\right)-I_{2}^{h} u\right\|_{0, \Gamma}
$$


and, from now on we focus our attention on the second term in the right hand side. By summing and subtracting the terms $\Pi(u)$ and $u_{\mid \Gamma}$, using the triangle inequality and the continuity of $\mathcal{S}_{2}$, we have:

$$
\left\|\mathcal{S}_{2}^{-1}\left(\Pi \mathcal{S}_{1}\left(I_{1}^{h} u\right)\right)-I_{2}^{h} u\right\|_{0, \Gamma} \leq C\left\{\left\|\Pi\left(\mathcal{S}_{1} I_{1}^{h} u\right)-\Pi(u)\right\|_{0, \Gamma}+\left\|\Pi(u)-u_{\mid \Gamma}\right\|_{0, \Gamma}+\left\|u_{\mid \Gamma}-\mathcal{S}_{2} I_{2}^{h} u\right\|_{0, \Gamma}\right\}
$$

By the $L^{2}$ continuity of the operators $\mathcal{S}_{1}$ and $\mathcal{S}_{2}$ and using the same step as in proof of Lemma 4.3 in order to bound the first and third terms in right hand side of (68), we end the proof.

\subsection{Consistency error}

In this section we analyze the contribution coming from the second term in the right hand side of (60) and we state the following proposition:

Proposition 4.5. Let $u \in H_{0}^{1}(\Omega)$ be the solution of the differential problem (52); we assume also $u \in H^{m}(\Omega) \cap$ $H_{0}^{1}(\Omega)$ for some $m>1$, then the following estimate holds for every $1 \leq s \leq\{k, m-1\}$ :

$$
\sup _{w_{h} \in \tilde{X}_{h}} \frac{\left(f, w_{h}\right)_{h}-a_{h}\left(u, w_{h}\right)}{\left\|w_{h}\right\|_{\star, h}} \leq C h^{s}\|u\|_{s+1, \Omega} .
$$

Proof of Proposition 4.5. Using the integration by parts and recalling that $u$ verifies (52), we have:

$$
\left(f, w_{h}\right)_{h}-a_{h}\left(u, w_{h}\right)=-\int_{\Gamma_{1}^{h}} \frac{\partial u}{\partial \mathbf{n}_{1}} w_{1, h} \mathrm{~d} \Gamma+\int_{\Gamma_{2}^{h}} \frac{\partial u}{\partial \mathbf{n}_{2}} w_{2, h} \mathrm{~d} \Gamma \quad \forall w_{h} \in \tilde{X}_{h}
$$

where we denote by $\mathbf{n}_{1}$ (resp. $\mathbf{n}_{2}$ ) the outer (resp. inner) normal to $\Omega_{1, h}\left(\right.$ resp. $\Omega_{2, h}$ ) at $\Gamma_{h}^{1}\left(\right.$ resp. $\left.\Gamma_{h}^{2}\right)$.

We apply the change of variables $\mathcal{S}_{1}$ (resp. $\mathcal{S}_{2}$ ) to the first (resp. second) integral in the right hand side of (70). Summing and subtracting the quantity $\int_{\Gamma} \mathcal{S}_{2}\left(\frac{\partial u}{\partial \mathbf{n}_{2}}\right) \mathcal{S}_{1} w_{1, h} \mathrm{~d} \Gamma$ and recalling the fact that the test function $w_{h}$ fulfills the constraint (56), we obtain that the following inequality holds for every $\varphi_{h} \in \tilde{M}_{h}$ :

$$
\begin{aligned}
\mid \int_{\Gamma_{1}^{h}} \frac{\partial u}{\partial \mathbf{n}_{1}} w_{1, h} \mathrm{~d} \Gamma-\int_{\Gamma_{2}^{h}} & \frac{\partial u}{\partial \mathbf{n}_{2}} w_{2, h} \mathrm{~d} \Gamma \mid \leq \int_{\Gamma}\left[\mathcal{S}_{1} \frac{\partial u}{\partial \mathbf{n}_{1}}-\mathcal{S}_{2} \frac{\partial u}{\partial \mathbf{n}_{2}}\right] \mathcal{S}_{1} w_{1, h} \mathrm{~d} \Gamma \\
& +\int_{\Gamma}\left[\mathcal{S}_{2}\left(\frac{\partial u}{\partial \mathbf{n}_{2}}\right)-\mathcal{S}_{2} \varphi_{h}\right]\left(\mathcal{S}_{1} w_{1, h}-\mathcal{S}_{2} w_{2, h}\right) \mathrm{d} \Gamma+C h^{k} \sum_{i=1}^{2}\left\|\frac{\partial u}{\partial \mathbf{n}_{i}}\right\|_{0, \Gamma_{i, h}}\left\|w_{i, h}\right\|_{0, \Gamma_{i, h}}
\end{aligned}
$$

where $\varphi_{h}$ is any function in $\tilde{M}_{h}$ and the last term, $C h^{k}$, comes from the fact that the Jacobian was neglected in the change of variables while writing the second integral in the right hand side.

We estimate now the two integrals in the right hand side of (71). Starting from the first one, the following estimate holds:

$$
\begin{aligned}
\left|\left[\mathcal{S}_{1}\left(\frac{\partial u}{\partial \mathbf{n}_{1}}\right)-\mathcal{S}_{2}\left(\frac{\partial u}{\partial \mathbf{n}_{2}}\right)\right] \mathcal{S}_{1} w_{1, h} d \Gamma\right| \leq & C\left\|\mathcal{S}_{1}\left(\frac{\partial u}{\partial \mathbf{n}_{1}}\right)-\mathcal{S}_{2}\left(\left.\frac{\partial u}{\partial \mathbf{n}_{1}}\right|_{\Gamma_{2, h}}\right)\right\|_{0, \Gamma}\left\|w_{1, h}\right\|_{0, \Gamma_{1, h}} \\
& +C\left\|\frac{\partial u}{\partial \mathbf{n}_{2}}-\left.\frac{\partial u}{\partial \mathbf{n}_{1}}\right|_{\Gamma_{2, h}}\right\|\left\|_{0, \Gamma_{2, h}}\right\| w_{1, h} \|_{0, \Gamma_{1, h}}
\end{aligned}
$$


Furthermore using the same argument used in (66) (resp. (65)) in (72) in the case $k=1$ (resp. $k>1$ ), we have:

$$
\begin{array}{ll}
\left\|\mathcal{S}_{1}\left(\frac{\partial u}{\partial \mathbf{n}_{1}}\right)-\mathcal{S}_{2}\left(\left.\frac{\partial u}{\partial \mathbf{n}_{1}}\right|_{\Gamma_{2, h}}\right)\right\|_{0, \Gamma} \leq C h\|u\|_{2, \Omega} & k=1 \\
\left\|\mathcal{S}_{1}\left(\frac{\partial u}{\partial \mathbf{n}_{1}}\right)-\mathcal{S}_{2}\left(\left.\frac{\partial u}{\partial \mathbf{n}_{1}}\right|_{\Gamma_{2, h}}\right)\right\|_{0, \Gamma} \leq C h^{k+1}\|u\|_{7 / 2, \Omega} & k>1 \\
\left\|\frac{\partial u}{\partial \mathbf{n}_{2}}-\left.\frac{\partial u}{\partial \mathbf{n}_{1}}\right|_{\Gamma_{2, h}}\right\|_{0, \Gamma_{2, h}} \leq\|u\|_{2, \Omega}\left\|\mathbf{n}_{1}-\mathbf{n}_{2}\right\|_{0, \Gamma_{2, h}} \leq C h^{k}\|u\|_{2, \Omega} & k \geq 1 .
\end{array}
$$

Using (73) in (72) we obtain for every $1 \leq s \leq \min \{k, m-1\}$

$$
\left|\int_{\Gamma_{1, h}}\left[\mathcal{S}_{1}\left(\frac{\partial u}{\partial \mathbf{n}_{1}}\right)-\mathcal{S}_{2}\left(\frac{\partial u}{\partial \mathbf{n}_{2}}\right)\right] \mathcal{S}_{1} w_{1, h} \mathrm{~d} \Gamma\right| \leq C h^{s}|| u \|_{s+1, \Omega} .
$$

For what concerns the second term in the right hand side of (71), we start by writing:

$$
\begin{array}{r}
\left|\int_{\Gamma}\left[\mathcal{S}_{2}\left(\frac{\partial u}{\partial \mathbf{n}_{2}}\right)-\mathcal{S}_{2} \varphi_{h}\right]\left(\mathcal{S}_{1} w_{1, h}-\mathcal{S}_{2} w_{2, h}\right) \mathrm{d} \Gamma\right| \leq C\left\{\inf _{\varphi_{h} \in \tilde{M}_{h}}\left\|\frac{\partial u}{\partial \mathbf{n}}-\mathcal{S}_{2} \varphi_{h}\right\|_{-1 / 2, \Gamma}\right. \\
\left.+\left\|\frac{\partial u}{\partial \mathbf{n}}-\mathcal{S}_{2} \frac{\partial u}{\partial \mathbf{n}_{2}}\right\|_{-1 / 2, \Gamma}\right\}\left\|w_{h}\right\|_{\star, h} .
\end{array}
$$

Now, the second term in the right hand side is treated with the same arguments as the ones used above in this proof, namely (73), while, by the Aubin-Nitsche argument, we obtain:

$$
\inf _{\varphi_{h} \in \tilde{M}_{h}}\left\|\frac{\partial u}{\partial \mathbf{n}}-\mathcal{S}_{2} \varphi_{h}\right\|_{-1 / 2, \Gamma} \leq h^{s}\|u\|_{s+1, \Omega} \quad 1 \leq s \leq \min \{k, m-1\} .
$$

Finally using estimates (74) and (75), we obtain that (69) holds.

Remark 4.6. In this section the matching condition and the definition of the approximation space (55-56) depend on the choice of the one-to-one shift operators $\mathcal{S}_{1}$ and $\mathcal{S}_{2}$ and of the integration domain in (56). Nevertheless we point out that other choices are possible and the associated proofs (which might use the explicit form of the one-to-one operator) still hold when replacing $\mathcal{S}_{i}$ by other regular one-to-one operators verifying condition (53). Moreover the interface condition can be replaced with a condition defined on $\Gamma_{i}^{h}$, for $i=1$ or $i=2$, or also, if needed, on any $\Gamma_{h}$ polygonal inscribed into $\Gamma$ whose vertices are a superset of the union of the vertices of $\Gamma_{1, h}$ and $\Gamma_{2, h}$. We set:

$$
\begin{array}{ll}
\mathcal{S}_{1,2}: L^{2}\left(\Gamma_{1}^{h}\right) \rightarrow L^{2}\left(\Gamma_{2}^{h}\right) & u \mapsto \mathcal{S}_{2}^{-1} \mathcal{S}_{1} u \\
\mathcal{S}_{2,1}: L^{2}\left(\Gamma_{2}^{h}\right) \rightarrow L^{2}\left(\Gamma_{1}^{h}\right) & \mathcal{S}_{2,1}=\mathcal{S}_{1,2}^{-1}
\end{array}
$$

The condition (56) could be replaced by any of the following ones:

$$
\begin{array}{ll}
\int_{\Gamma_{2}^{h}}\left(\mathcal{S}_{1,2} v_{1, h}\left(r_{-t} \mathbf{x}\right)-v_{2, h}(\mathbf{x})\right) \varphi_{h}(\mathbf{x}) \mathrm{d} \Gamma=0 & \forall \varphi_{h} \in \tilde{M}_{h} ; \\
\int_{\Gamma_{1}^{h}}\left(v_{1, h}\left(r_{-t} \mathbf{x}\right)-\mathcal{S}_{2,1} v_{2, h}(\mathbf{x})\right) \mathcal{S}_{2,1} \varphi_{h}(\mathbf{x}) \mathrm{d} \Gamma=0 & \forall \varphi_{h} \in \tilde{M}_{h} ; \\
\int_{\Gamma_{h}} \mathcal{S}_{h}\left(\mathcal{S}_{1} v_{1, h}\left(r_{-t} \mathbf{x}\right)-\mathcal{S}_{2} v_{2, h}(\mathbf{x})\right) \mathcal{S}_{h} \mathcal{S}_{2} \varphi_{h}(\mathbf{x}) \mathrm{d} \Gamma=0 & \forall \varphi_{h} \in \tilde{M}_{h} ;
\end{array}
$$


where in the last line the operator $\mathcal{S}_{h}$ is then associated to the shift operator from $\Gamma$ to $\Gamma_{h}$. From a theoretical point of view the difference among these constraints relies on changes of the integration variables where Jacobians can be neglected thanks to (53). From a numerical point of view it brings flexibility to be able to deal with other choices than the ones specifically used in the proofs of the previous sections.

Remark 4.7. In this section we carried out the estimates of the best approximation and consistency errors for the mortar element method in the case of iso-parametric finite elements. Although we explicitly treated only the case of interest, that is a decomposition in only two sub-domains with the interface $\Gamma$ which is a manifold without boundary, the proofs we presented apply unchanged to more general decompositions. The use of isoparametric finite elements preserves then the optimality of the mortar method and make the assembly of the mass and stiffness matrix inexpensive.

\subsection{Error estimate in the case of iso-parametric finite elements}

The theory developed in this section can of course be applied for the discretisation of problem (8) and allow the use of iso-parametric finite elements along the interface $\Gamma$.

Applying the machinery developed in Sections 2 and 3 with this new choice of approximation space, we obtain the following Theorem:

Theorem 4.8. Let

$\tilde{\mathcal{U}}_{h}^{n}:=\left\{v_{h} \in \tilde{X}_{h}\right.$ such that $v_{h \mid \partial \Omega}=0$ and $\left.\int_{\Gamma}\left(\mathcal{S}_{1} v_{1, h}\left(r_{-n} \mathbf{x}\right)-\mathcal{S}_{2} v_{2, h}(\mathbf{x})\right) \mathcal{S}_{2} \varphi_{h}(\mathbf{x}) \mathrm{d} \Gamma=0 \quad \forall \varphi_{h} \in \tilde{M}_{h}\right\}$ and $\left\{\tilde{u}_{h}^{n}\right\}_{n}$ be the solution of the problem

$$
\begin{aligned}
& \forall n=0, . ., N-1, \quad \text { find } \tilde{u}_{h}^{n+1} \in \tilde{\mathcal{U}}_{h}^{n+1} \text { such that: } \\
& \qquad \int_{\mathcal{C}} \sigma \frac{\tilde{u}_{h}^{n+1}-\tilde{u}_{h}^{n}}{\delta t} v_{h}^{n+1} \mathrm{~d} \Omega+a_{h}\left(\tilde{u}_{h}^{n+1}, v_{h}^{n+1}\right)=\left(J_{s}^{n+1}, v_{h}^{n+1}\right)_{\Omega_{2}} \quad \forall v_{h}^{n+1} \in \tilde{\mathcal{U}}_{h}^{n+1} .
\end{aligned}
$$

If the solution $u$ of the problem (8) verifies the same regularity assumptions as in Theorem 3.2, the following optimal error estimate holds:

$$
\begin{aligned}
\left\|\sqrt{\sigma}\left(u^{n}-\tilde{u}_{h}^{n}\right)\right\|_{0, \mathcal{C}}^{2}+ & \alpha \sum_{i=1}^{n} \delta t \sum_{j=1}^{2}\left\|u_{j}^{i}-\tilde{u}_{j, h}^{i}\right\|_{1, \Omega_{j} \cap \Omega_{j, h}}^{2} \leq C(T, \sigma, \mu)\left\{h^{2 s} \sum_{i=1}^{n} \delta t\left|u^{i}\right|_{\star, s+1}^{2}\right. \\
& \left.+h^{2(s+1)}\left(\left\|u^{n}\right\|_{s+1, \mathcal{C}}^{2}+\left\|u^{0}\right\|_{s+1, \mathcal{C}}^{2}\right)+(\delta t)^{2}\left\|\frac{\partial^{2} u}{\partial t^{2}}\right\|_{L^{2}\left(0, T ; L^{2}(\mathcal{C})\right)}^{2}+h^{2 s}\left\|\frac{\partial u}{\partial t}\right\|_{L^{2}\left(0, T ; \mathcal{H}^{s}(\Omega)\right)}^{2}\right\}
\end{aligned}
$$

for all $n=1, . ., N$ and where the constant $C(T, \sigma, \mu)$ depends on the parameters of the problem, but neither on St nor on $h$.

\section{IMPLEMENTATION ASPECTS}

In this section we detail the some aspects related to the implementation of the proposed method (26).

We focus our attention, firstly, on the numerical treatment of the interface condition (25) which appears to be a new task in a standard finite element code and, secondly, on the construction of the final system. To simplify the exposition, we treat the case in which the rotor doesn't move but the same arguments hold also when the rotor moves and any difference will be pointed out where necessary. 
In Section 4 it is proved that the use of curved triangulations can be avoided and, without loss of generality, we assume here that:

- $\Omega_{1}$ and $\Omega_{2}$ are decomposed by triangular meshes with straight edges and non-matching at the interface $\Gamma$.

- The degree of the polynomials is set equal to 1 in both domains.

\subsection{Numerical treatment of the mortar condition}

Since we use standard triangulation on both domains, the mortar condition must be treated according to Section 4. In the following we focus our attention on the implementation of the constraint (56) and of some of its variants (see Rem. 4.6). Below, in Figure 1, we present the general configuration we have to deal with from the implementation point of view: on the left, a simplified situation with only one triangle from the stator and rotor meshes and, on the right, an enlargement of a global mesh in a neighbourhood of the interface. As it can be seen, the stator mesh penetrates that of the rotor and vice versa.
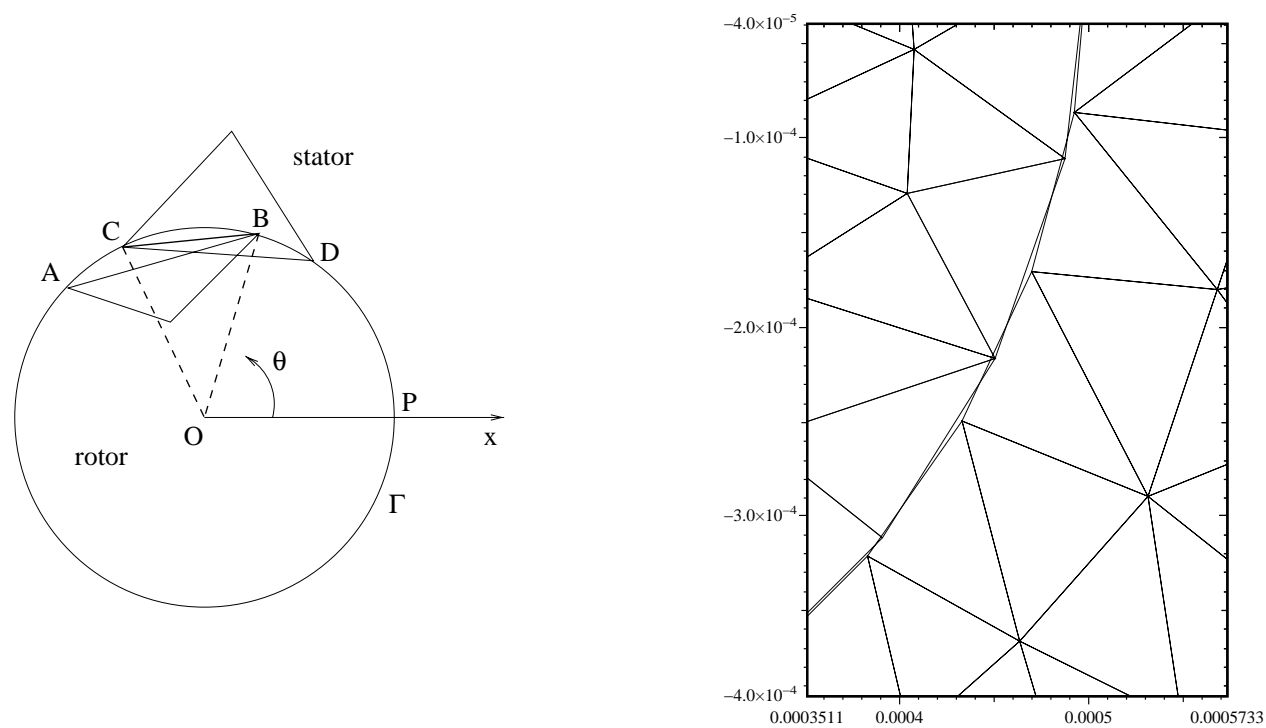

Figure 1. Intersection between two mesh sides belonging one to a stator mesh triangle and the other to a rotor one (left). A real mesh configuration in a neighbourhood of the interface (right). The intersection of the two mesh edges $A B$ and $C D$ can be defined either in terms of angles or in terms of segments. In the case of the figure, the intersection is represented either by the interval $\left(\theta_{B}, \theta_{C}\right)$ or by the line $C B$.

We propose two different approaches to compute the integral in the mortar constraint.

1. The first approach is based on the representation of the mortar constraint presented in Section 4 and on a clever choice of the operators $S_{i}$. We choose $S_{i}$ in a way that the functions involved in the integral (56) are piecewise polynomials of first degree with respect to the curvilinear parameter $s$.

2. The second one relies on the representation (79) of the mortar constraint and $\Gamma_{h}$ is chosen to be the polygonal obtained by using all the stator and rotor mesh nodes lying on $\Gamma$. In this approach a quadrature formula is used.

In what follows, keeping in mind Figure 1 (left), we will denote by $\varphi_{A}^{1}$ and $\varphi_{B}^{1}$ the basis functions associated to nodes $A$ and $B$ of the rotor mesh triangle and by $\varphi_{C}^{2}$ and $\varphi_{D}^{2}$ those associated to nodes $C$ and $D$ of the stator mesh triangle. 


\section{First approach}

Since $\Gamma$ a circle, we know a global parameterization of it and the integration of polynomials of degree two over $\Gamma$ can be done exactly. In the following we see that through a suitable choice of the operators, the mortar constraint can involve only integrals of that type. We use the constraint (56) and we choose the operators $S_{i}$ in such a way that $\mathcal{S}_{i}$ send the set of piecewise linear functions onto itself. Namely the operators $\mathcal{S}_{i}$ send segments of equal length into segments of equal length. In this way, referring to Figure 1 for the considered configuration, we have the following expressions

$$
\begin{array}{ll}
\mathcal{S}_{1}\left(\varphi_{A}^{1}\right)(s)=\frac{s_{B}-s}{s_{B}-s_{A}}, & \mathcal{S}_{1}\left(\varphi_{B}^{1}\right)(s)=\frac{s-s_{A}}{s_{B}-s_{A}}, \quad \text { on the } \operatorname{arc} A B ; \\
\mathcal{S}_{2}\left(\varphi_{C}^{2}\right)(s)=\frac{s_{D}-s}{s_{D}-s_{C}}, \quad \mathcal{S}_{2}\left(\varphi_{D}^{2}\right)(s)=\frac{s-s_{C}}{s_{D}-s_{C}}, \quad \text { on the } \operatorname{arc} C D .
\end{array}
$$

The idea is that now the integrands in (56) are product of piecewise polynomials of degree one.

This approach relies on the intersection between the two discretizations of the interface given in terms of angles and the general integral that one has to calculate is:

$$
\int_{\operatorname{arc} B C} \mathcal{S}_{1}\left(\varphi_{B}^{1}\right) \mathcal{S}_{2}\left(\varphi_{C}^{2}\right) \mathrm{d} s=\int_{\theta_{B}}^{\theta_{C}}\left(\frac{\theta-\theta_{A}}{\theta_{B}-\theta_{A}}\right)\left(\frac{\theta_{D}-\theta}{\theta_{D}-\theta_{C}}\right) \rho \mathrm{d} \theta
$$

that can be exactly computed $(\rho=|O-P|)$.

\section{Second approach}

In this second approach we use a constraint of the type (79) where $\Gamma_{h}$ is the polygonal inscribed in $\Gamma$ composed of all the intersection segments. Its nodes are all the stator and rotor mesh nodes lying $\Gamma$. The operator $S_{i}$ and $S_{h}$ are chosen to be the orthogonal projection with respect to $\Gamma_{i, h}$.

If we look at Figure 1, the integral we are considering as example becomes

$$
\int_{\operatorname{seg} A B \cap \operatorname{seg} C D} \varphi_{B}^{1} \varphi_{C}^{2} \mathrm{~d} t
$$

This approach relies on the intersection between the two discretizations of the interface given in terms of segments as it has been previously defined. If the intersection segment $C B$ is not an empty set, we have

$$
\int_{\operatorname{seg} A B \cap \operatorname{seg} C D} \varphi_{B}^{1} \varphi_{C}^{2} \mathrm{~d} t=\sum_{k \mid \xi_{k} \in \operatorname{seg} C B} \varphi_{B}^{1}\left(x_{k}\right) \varphi_{C}^{2}\left(y_{k}\right) \omega_{k}
$$

where $x_{k}$ and $y_{k}$ are the orthogonal projections of the integration nodes $\xi_{k}$ on $A B$ and $C D$ respectively and $\omega_{k}$ are the weights of the integration formula.

\subsection{The construction of the final matrix system}

In this section, we aim at writing problem (26) in a matrix form. First, we construct a basis of the approximation space $\mathcal{U}_{h}^{t}$. It involves a set of linear systems whose solutions represent the columns of a matrix, called $Q$ in the what follows, that allows to couple at the interface the information coming from the stator and rotor domains. Its construction is a crucial point in the method implementation. Next, we present the matrix structure of the discretized problem pointing out the characteristics of the involved matrix. 
It is important to remark that all the line integrals over $\Gamma$ which appear in what follows, have to be considered as explained in the previous section, although by abuse of notation we shall use the symbol $\int_{\Gamma}$ to simplify the description.

\subsubsection{A basis for the approximation space $\mathcal{U}_{h}^{t}$}

Let us consider the discrete spaces $X_{i, h_{i}}$ defined in $(22)$ with $k_{1}=k_{2}=1$. The degrees of freedom are the values at the vertices of the mesh triangles $K$. We denote the nodes of triangle $K$ by $\mathbf{a}_{K}$ and we define the sets of global nodes $\Xi_{i}^{0}$

$$
\Xi_{i}^{0}:=\left\{\mathbf{a}_{K} \mid K \in \mathcal{T}_{i, h_{i}}, \mathbf{a}_{K} \notin \partial \Omega_{i} \backslash \Gamma\right\}
$$

In defining the set $\Xi_{h}^{0}$, an assembly process has taken place and in the global numeration we denote a node by a. The two sets of nodes on the interface will be denoted as follows

$$
\xi^{i}=\left\{\mathbf{a} \in \Xi_{i}^{0} \cap \Gamma\right\}
$$

and we define $m_{\Gamma}^{i}=\operatorname{card}\left(\xi^{i}\right)$ and $m^{i}=\operatorname{card}\left(\Xi_{i}^{0}\right)$.

Let us denote by $\varphi_{\mathbf{a}}^{i}$ the basis function associated to node $\mathbf{a} \in \Xi_{i}^{0}$. This function is the element of the spaces $X_{i, h_{i}}$ defined by

$$
\varphi_{\mathbf{a}}^{i}(\mathbf{b})=\delta_{\mathbf{a b}} \quad \forall \mathbf{b} \in \Xi_{i}^{0}
$$

where $\delta_{\mathbf{a b}}$ is the Kronecker symbol.

Let us denote by $\mathcal{B}$ a basis for $\mathcal{U}_{h}^{0}(t)$ : we suppose that

$$
\mathcal{B}=\mathcal{B}^{1} \cup \mathcal{B}^{2} \cup \mathcal{B}^{\Gamma}
$$

where the sets of functions $\mathcal{B}^{1}, \mathcal{B}^{1}$, and $\mathcal{B}^{\Gamma}$ will be defined below.

The functions in $\mathcal{B}^{1}$ are zero in $\overline{\Omega_{2}}$, the functions in $\mathcal{B}^{2}$ are zero in $\overline{\Omega_{1}}$, and the functions in $\mathcal{B}^{\Gamma}$ have their support in the union of the mortar elements. The mortar elements are the triangles having a vertex lying on $\Gamma$.

Due to the properties of the considered basis functions, we set

$$
\begin{aligned}
& \mathcal{B}^{1}=\left\{\left(\varphi_{\mathbf{a}}^{1}, 0\right) \mid \mathbf{a} \in \Xi_{1}^{0} \backslash \xi^{1}\right\}, \\
& \mathcal{B}^{2}=\left\{\left(0, \varphi_{\mathbf{a}}^{2}\right) \mid \mathbf{a} \in \Xi_{2}^{0} \backslash \xi^{2}\right\}
\end{aligned}
$$

and a possible choice for the basis functions centered on the nodes lying on the interface is

$$
\mathcal{B}^{\Gamma}=\left\{\left(\varphi_{\mathbf{a}}^{1}, \sum_{\mathbf{b} \in \xi^{2}} q_{\mathbf{b}}^{\mathbf{a}} \varphi_{\mathbf{b}}^{2}\right) \mid \mathbf{a} \in \xi^{1}, q_{\mathbf{b}}^{\mathbf{a}} \in \mathbb{R}\right\} .
$$

For each of the $m_{\Gamma}^{1}$ nodes $\mathbf{a}$, the coefficients $q_{\mathbf{b}}^{\mathbf{a}}, \mathbf{b} \in \xi^{2}$ are determined by imposing the integral matching condition

$$
\int_{\Gamma}\left(\varphi_{\mathbf{a}}^{1}-\sum_{\mathbf{b} \in \xi^{2}} q_{\mathbf{b}}^{\mathbf{a}} \varphi_{\mathbf{b}}^{2}\right) \varphi_{\mathbf{c}}^{2} \mathrm{~d} \Gamma=0 \quad \forall \mathbf{c} \in \xi^{2}
$$

Each of these $m_{\Gamma}^{1}$ systems of $m_{\Gamma}^{2}$ equations can be put into the following matrix form

$$
C \mathbf{q}^{i}=\mathbf{D}^{i}
$$


where

$$
\begin{gathered}
\left(\mathbf{q}^{i}\right)^{T}=\left(q_{\mathbf{b}_{1}}^{\mathbf{a}_{i}}, \ldots, q_{\mathbf{b}_{m_{\Gamma}}}^{\mathbf{a}_{i}}\right) \\
C(r, j)=\int_{\Gamma} \varphi_{\mathbf{a}_{r}}^{2} \varphi_{\mathbf{a}_{j}}^{2} \mathrm{~d} \Gamma \quad r, j: \mathbf{a}_{r}, \mathbf{a}_{j} \in \xi^{2}, \\
\mathbf{D}^{i}(j)=\int_{\Gamma} \varphi_{\mathbf{a}_{i}}^{1} \varphi_{\mathbf{a}_{j}}^{2} \mathrm{~d} \Gamma \quad i: \mathbf{a}_{i} \in \xi^{1}, j: \mathbf{a}_{j} \in \xi^{2} .
\end{gathered}
$$

The system (81) allows us to determine the vector $\mathbf{q}^{i}, \forall i: \mathbf{a}_{i} \in \xi^{1}$, and thus the set of basis functions on the interface. For following considerations, it is useful to define the rectangular matrix $Q$ of dimension $m_{\Gamma}^{2} \times m_{\Gamma}^{1}$

$$
Q=C^{-1} D
$$

\subsubsection{The matrix structure of the discretized problem}

The system we solve to get numerical results can be obtained directly by expanding $u_{h}=\left(u_{1, h}, u_{2, h}\right)$ in terms of the basis functions of $\mathcal{B}$ and inserting this expansion in equation (26).

Here we prefer to get the final system with a different two-step procedure: the first step consists in writing, in each domain, the system associated to equation (26) with homogeneous Neumann conditions on the interface $\Gamma$. In the second step the two previous systems are coupled by means of the mortar condition. This procedure is easier to be understood and involves few modifications in an already existing finite element code. Now, let us go through it in a more detailed way.

We expand the numerical approximation $u_{h}=\left(u_{1, h}, u_{2, h}\right)$ of the solution $u$ in terms of the basis functions of $X_{i, h_{i}}$ :

$$
\begin{aligned}
& u_{1, h}(\mathbf{x}, t)=\sum_{i: \mathbf{a}_{i} \in \Xi_{1}^{0}} u_{i}^{1}(t) \varphi_{\mathbf{a}_{i}}^{1}(\mathbf{x}), \\
& u_{2, h}(\mathbf{x}, t)=\sum_{r: \mathbf{a}_{r} \in \Xi_{2}^{0}} u_{r}^{2}(t) \varphi_{\mathbf{a}_{r}}^{2}(\mathbf{x}),
\end{aligned}
$$

where we have used $m^{1}$ coefficients $u_{i}^{1}$ and $m^{2}$ coefficients $u_{r}^{2}$. Among these $m^{1}+m^{2}$ coefficients, only card $\mathcal{B}=m^{1}+m^{2}-m_{\Gamma}^{2}$ are real unknowns. In fact, the $m_{\Gamma}^{1}$ coefficients associated to nodes in $\xi^{1}$ are linked to the $m_{\Gamma}^{2}$ coefficients associated to nodes in $\xi^{2}$ through the integral matching condition (25).

We divide the unknowns in each domain in two blocks with names reported in parentheses: the first block $\left(\mathbf{u}_{\Gamma}\right)$ contains the unknowns associated to the $m_{\Gamma}^{j}$ nodes lying on $\Gamma$ and the second block $\left(\mathbf{u}_{i}\right)$ those associated to the $m^{j}-m_{\Gamma}^{j}$ nodes interior to the domain $(j=1,2)$. The vectors of unknowns are then

$$
\mathbf{u}^{1}=\left(\mathbf{u}_{\Gamma}^{1}, \quad \mathbf{u}_{i}^{1}\right)^{T}, \mathbf{u}^{2}=\left(\begin{array}{cc}
\mathbf{u}_{\Gamma}^{2}, & \mathbf{u}_{i}^{2}
\end{array}\right)^{T}
$$

where the exponent ${ }^{T}$ stands for the transpose operator. In each domain, we build the system associated to equation (26) with a homogeneous Neumann condition at the interface $\Gamma$. In this way, we obtain two linear systems of the following form $(j=1,2)$

$$
A^{j}\left(\begin{array}{c}
\mathbf{u}_{\Gamma}^{j} \\
\mathbf{u}_{i}^{j}
\end{array}\right)_{t=t_{n+1}}=\left(\begin{array}{c}
\mathbf{F}_{\Gamma}^{j} \\
\mathbf{F}_{i}^{j}
\end{array}\right)_{t=t_{n}} \text { with } A^{j}=\left(\begin{array}{cc}
\frac{M_{\Gamma, \Gamma}^{j}}{\Delta t}+K_{\Gamma, \Gamma}^{j} & \frac{M_{\Gamma, i}^{j}}{\Delta t}+K_{\Gamma, i}^{j} \\
\frac{M_{i, \Gamma}^{j}}{\Delta t}+K_{i, \Gamma}^{j} & \frac{M_{i, i}^{j}}{\Delta t}+K_{i, i}^{j}
\end{array}\right) .
$$


Neither $\mathbf{u}^{1}$ nor $\mathbf{u}^{2}$ are solution of the previous problems since the two sets of values $\mathbf{u}_{\Gamma}^{1}$ and $\mathbf{u}_{\Gamma}^{2}$ are linked one to the other by the mortar matching condition. Choosing the traces on $\Gamma$ of the stator shape functions as mortar elements, we have that

$$
Q_{n+1} \mathbf{u}_{\Gamma}^{1}\left(t_{n+1}\right)=\mathbf{u}_{\Gamma}^{2}\left(t_{n+1}\right), \quad Q_{n} \mathbf{u}_{\Gamma}^{1}\left(t_{n}\right)=\mathbf{u}_{\Gamma}^{2}\left(t_{n}\right)
$$

where $Q_{n+1}$ and $Q_{n}$ are the rectangular full matrices of dimension $m_{\Gamma}^{2} \times m_{\Gamma}^{1}$ obtained from the matching condition discretization at time $t_{n+1}$ and $t_{n}$. We introduce then the following matrices:

$$
\tilde{Q}_{n+1}=\left(\begin{array}{ccc}
0 & Q_{n+1} & 0 \\
I d & 0 & 0 \\
0 & I d & 0 \\
0 & 0 & I d
\end{array}\right) \quad \text { and } \quad A=\left(\begin{array}{cc}
A^{2} & 0 \\
0 & A^{1}
\end{array}\right)
$$

together with $\tilde{Q}_{n}$ of the same structure as $\tilde{Q}_{n+1}$ but involving $Q_{n}$ and

$$
M=\left(\begin{array}{cc}
M^{2} & 0 \\
0 & M^{1}
\end{array}\right) \quad \text { where } \quad M^{j}=\left(\begin{array}{cc}
M_{\Gamma, \Gamma}^{j} & M_{\Gamma, i}^{j} \\
M_{i, \Gamma}^{j} & M_{i, i}^{j}
\end{array}\right) .
$$

Indicating by $\mathbf{w}_{n+1}$ the independent unknowns at time $t_{n+1}$, i.e.

$$
\mathbf{w}_{n+1}=\left(\mathbf{u}_{i}^{2}\left(t_{n+1}\right), \quad \mathbf{u}_{\Gamma}^{1}\left(t_{n+1}\right), \quad \mathbf{u}_{i}^{1}\left(t_{n+1}\right)\right)^{T}
$$

the approximation of the physical solution is computed solving the system

$$
\tilde{Q}_{n+1}^{T} A \tilde{Q}_{n+1} \mathbf{w}_{n+1}=\tilde{Q}_{n+1}^{T} \frac{M}{\Delta t} \tilde{Q}_{n} \mathbf{w}_{n}+\tilde{Q}_{n+1}^{T} \mathbf{J}_{n+1} .
$$

In particular, the left-hand side matrix can be represented in a block-displayed structure as follows

$$
\tilde{Q}_{n+1}^{T} A \tilde{Q}_{n+1}=\left(\begin{array}{ccc}
A_{i, i}^{2} & A_{i, \Gamma}^{2} Q_{n+1} & 0 \\
Q_{n+1}^{T} A_{\Gamma, i}^{2} & Q_{n+1}^{T} A_{\Gamma, \Gamma}^{2} Q_{n+1}+A_{\Gamma, \Gamma}^{1} & A_{\Gamma, i}^{1} \\
0 & A_{i, \Gamma}^{1} & A_{i, i}^{1}
\end{array}\right)
$$

The final matrix is symmetric, since for both domains we have that $A_{i, \Gamma}=\left(A_{\Gamma, i}\right)^{T}$, and positive. The final system can be solved either by a direct method such as the Cholesky factorization or by an iterative one such as the Conjugate Gradient procedure [23]. In the second case, residuals can be computed in parallel thanks to the two-step procedure that has been chosen to derive the stiffness and mass matrices (see [4]).

For what concerns the way the mortar and the final system matrices are built, there is no difference in structure between the case in which the rotor domain is fixed and the case with the rotor domain rotating around its center. In the first case, the matrix $Q$ is constructed once at the exterior of the temporal loop. In the second case, it is built at each time step: the intersection between the stator and rotor meshes at the interface changes from one time iteration to the next one and so the elements of $Q$ do. The stiffness and the mass matrices in $A$ and $M$, instead, do not depend on time. 


\section{Numerical RESUlts}

In the following, we are going to present some results concerning the comparison between the two techniques adopted for the matching condition discretization and the ability of the method at reproducing the physical solution (continuous at the interface). Moreover, we will analyse the precision of the method and present the results related to its application in studying a more realistic, even if academic, eddy currents problem (TE formulation is here considered).

\subsection{Geometry and simulation parameters}

We consider the situation displayed in Figure 2 (left). The stator is a square of edge $L=2 \mathrm{~mm}$, and the rotor is a circle of radius $\rho=0.5 \mathrm{~mm}$ and center at the origin of the $(x, y)$ coordinates system.
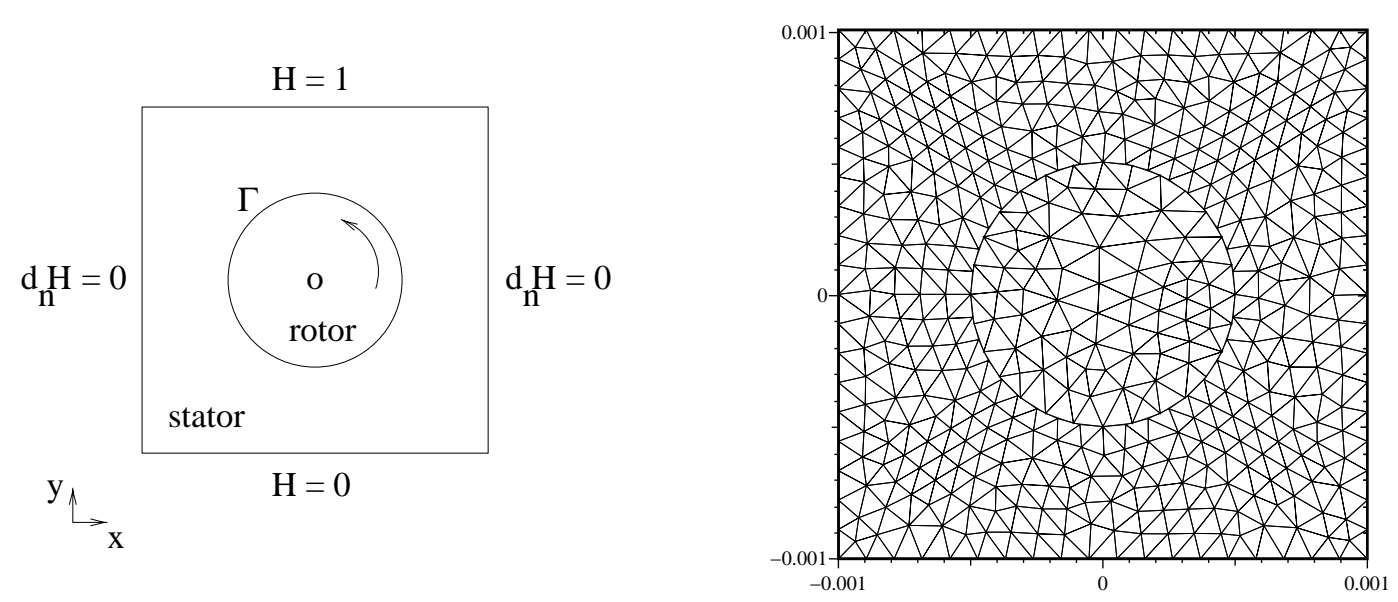

FiguRE 2. The geometry of the problem and boundary conditions (left); an example of stator and rotor meshes that do not match at the interface (right).

The source is represented by a constant magnetic field parallel to the $z$-axis, of intensity $0 \mathrm{~T} / \mathrm{m}$ at the bottom and $1 \mathrm{~T} / \mathrm{m}$ at the top of the stator domain; homogeneous Neumann conditions are considered on the stator vertical boundaries.

We use linear finite elements for the spatial discretization. The stator mesh is composed of 423 nodes and 736 triangles whereas the rotor one has 84 nodes and 138 triangles (see Fig. 2 (right); the mesh parameter $h$ is equal to $0.1 \mathrm{~mm}$ for both meshes.) A first order implicit Euler scheme is applied to discretize the temporal derivative.

A characteristic dimension of the problem is the depth of penetration of the magnetic field in the domain

$$
\delta=\frac{1}{\sqrt{\pi \mu_{0} \sigma f}}
$$

If $v$ is the rotation tangential speed, the rotation angular speed is $\omega=\frac{v}{\rho}$ to which it corresponds a frequency $f=\frac{v}{2 \pi \rho}$. The value $v=v_{1} \approx\left(18 \pi \rho 10^{2}\right)^{-1}$ has been chosen by supposing that

$$
\sigma=10^{9} \mathrm{~S} / \mathrm{m}, \quad \mu_{0}=4 \pi 10^{-7} \mathrm{H} / \mathrm{m}, \quad \delta \approx 3 \rho .
$$


To have the rotor cycling once in 40 time iterations, we impose that the rotation angle at each time step is equal to $9^{\circ}$. In this case we have

$$
\delta t=\delta t_{1}=\frac{9 \pi}{180 \omega_{1}} \approx 0.025 \mathrm{~s} .
$$

Finally, to accelerate the convergence of the simulation, we have initialized the solution of the magnetodynamic problem (2) with the solution of the magnetostatic one described by equation (2) without the time derivative term.

\subsection{Comparing the two approaches}

In Figure 3 are reported the results of the comparison among the two techniques we have adopted to numerically compute the line integrals involved in the mortar matrix construction. Results are obtained on the mesh displayed in Figure 2, with time step $\delta t=\delta t_{1}$ and supposing the rotor still. Each mesh node on the interface is located by its angle (reported on the $x$-axis). On the $y$-axis, we display the values of the computed solution at these nodes. Let us remark that on the interface we have at our disposal two sets of nodes: that of the nodes belonging to the stator mesh and that of the nodes belonging to the rotor mesh. In this case, we have reported the values at the stator mesh nodes: those related to the rotor mesh nodes describe the same curves (as it will be remarked in the next section).

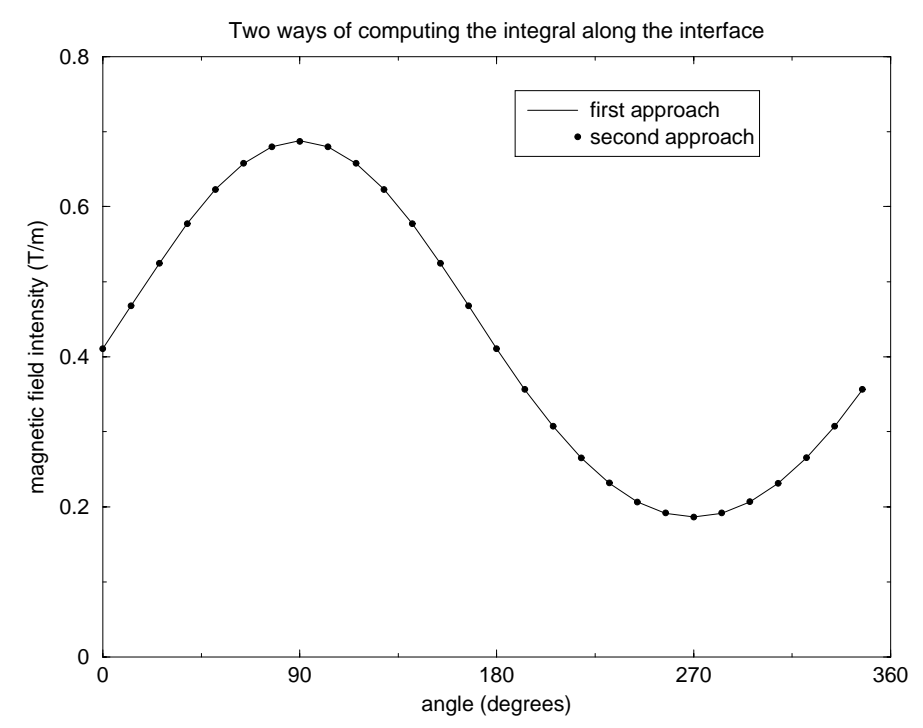

FIGURE 3. Values of the solution at all the stator mesh nodes on the interface when the elements of the mortar matrix are computed by the two considered techniques.

By looking at the Figure 3, we can see that the two approaches can not be distinguished from the point of view of the numerical results. Moreover, results confirm that the two approaches have the same order of approximation as proved in the theory.

\subsection{Comparing the stationary and the non-stationary cases}

In Figure 4, are shown the values of the computed solution at the stator and rotor mesh nodes lying on the interface. Once again, results are obtained on the mesh of Figure 2 with time step $\delta t=\delta t_{1}$ : the rotor is supposed still in one case and moving at speed $v_{1}$ in the second case. 


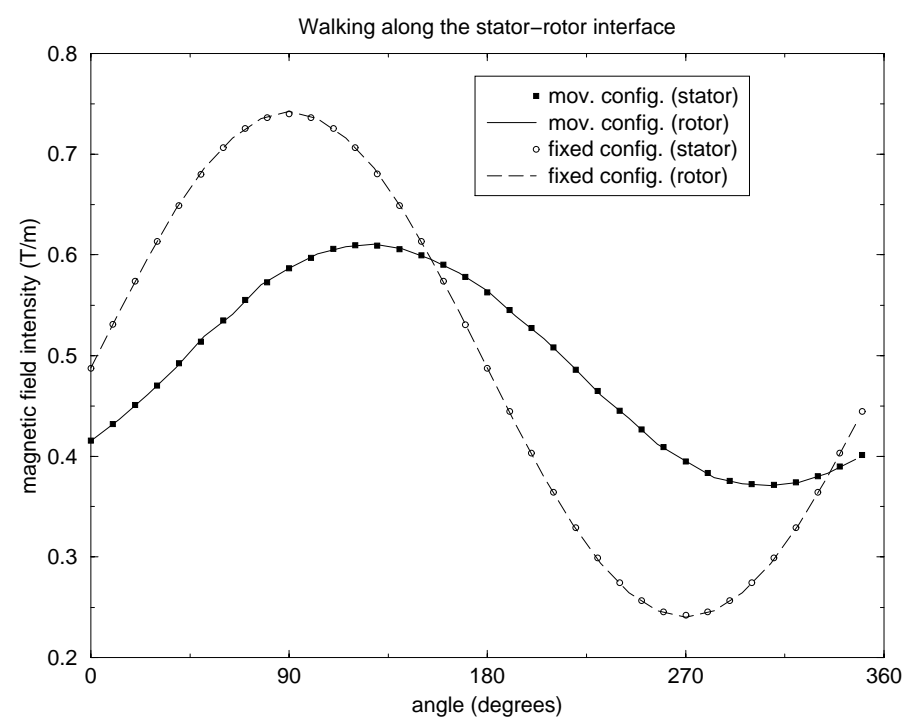

Figure 4. Values of the solution at all the nodes on the interface: the values are computed keeping once the rotor fixed and once rotating.

Despite the weak imposition of the matching condition, the results show that the numerical solution reproduces a physical one: indeed it looks "continuous" at the interface. Moreover, this qualitative "continuity" is respected even when the rotor domain is rotating around its center. The intensity of the computed magnetic field at the interface nodes (and in all the other rotor mesh nodes) is lower when the rotor is moving due to the minor penetration of the magnetic field in the moving part (any increase in the rotation speed $v$ implies a decrease in the depth of penetration $\delta$.)

\subsection{Precision of the method}

In this subsection we are going to analyse the precision of the proposed method and the simulation parameters, slightly different with those defined in the first section, will be defined later on. Here, we are going to make a quantitative comparison, at the interface $\Gamma$, between the "exact solution" $(u)$ and the one $\left(u_{h}\right)$ numerically computed with different meshes and different time steps. The term "exact solution" will refer either to the analytical solution when available (cases 1 and 2 in what follows) or to a solution numerically computed on the finest mesh with the smallest time step (case 3). The spatial error at a fixed instant $t$ is represented by

$$
\frac{\left\|u-u_{h}\right\|_{L^{2}(\Gamma)}}{\|1\| L_{L^{2}(\Gamma)}} \approx\left(\frac{\sum_{\mathbf{a}_{k} \in \Gamma}\left|\mathbf{a}_{k+1}-\mathbf{a}_{k}\right|\left(u-u_{h}\right)^{2}\left(\mathbf{a}_{k}, t\right)}{\sum_{\mathbf{a}_{k} \in \Gamma}\left|\mathbf{a}_{k+1}-\mathbf{a}_{k}\right|}\right)^{1 / 2}
$$

where $\mathbf{a}_{k}$ are nodes either belonging to $\mathcal{T}_{1, h_{1}}^{\Gamma}$ or to $\mathcal{T}_{2, h_{2}}^{\Gamma}$. In the first case, $u_{h}=u_{1, h_{1}}$, and in the second case, $u_{h}=u_{2, h_{2}}$. The temporal error is the maximum for $t \in[0, T]$ of the spatial errors. In the figures which follow, we will draw the logarithm of the spatial error with respect to the logarithm of the number $N$ of mesh nodes on $\Gamma$ and the temporal error with respect to the time step (in seconds) with a little difference in the last case. In the following we set $l=L / 2$. 
To spatially discretize the domain, we now consider different meshes such that the corresponding discretizations of the interface $\Gamma$ are nested: this means that $(i=1,2)$

$$
\mathcal{T}_{i, h_{i}^{r}}^{\Gamma} \subset \mathcal{T}_{i, h_{i}^{r+1}}^{\Gamma} \quad \text { where } \quad h_{i}^{r}=\frac{h_{i}}{2^{r}} \quad \text { and } \quad r=0,1,2,3,4
$$

and $\Gamma$ has successively 12, 24, 48, 96, 192 nodes. The number of nodes lying on $\Gamma$ is denoted by $N$. In the following, we will use the term "matching grid" to denote a couple of meshes $\mathcal{T}_{i, h_{i}}^{\Gamma}(i=1,2)$ such that $\mathcal{T}_{1, h_{1}}^{\Gamma}=\mathcal{T}_{2, h_{2}}^{\Gamma}$ and the term "non-matching grid" to denote a couple of meshes such that $\mathcal{T}_{1, h_{1}}^{\Gamma} \neq \mathcal{T}_{2, h_{2}}^{\Gamma}$. For all values of $r$, the defined meshes are matching grids: starting from these meshes, we obtain a couple of non-matching grids by simply rotating $\mathcal{T}_{1, h_{1}^{r}}^{\Gamma}$ with respect to $\mathcal{T}_{2, h_{2}^{r}}^{\Gamma}$ of a suitable angle.

The method precision will be tested in the three following cases:

(Case 1) on a magnetostatic problem;

(Case 2) on a magnetodynamic problem when the rotor does not move;

(Case 3) on a magnetodynamic problem when the rotor moves with a constant angular speed.

(Case 1): let us start by considering the magnetostatic problem described by equation (2) without the time derivative term. In this case, we suppose that the source, always represented by a constant magnetic field parallel to the $z$-axis, has intensity $-1 \mathrm{~T} / \mathrm{m}$ at the bottom and $1 \mathrm{~T} / \mathrm{m}$ at the top of the stator domain; homogeneous Neumann conditions are considered on the stator vertical boundaries. Due to these assumptions, the magnetic field $\mathbf{H}=(0,0, \mathrm{H})$ depends only on the $y$ coordinate and is solution of the following problem

$$
\frac{\mathrm{d}^{2} \mathrm{H}}{\mathrm{d} y^{2}}=0, \quad \mathrm{H}(l)=1, \quad \mathrm{H}(-l)=-1 .
$$

So, the (third component of the) analytical (exact) solution of the problem is given by $\mathrm{H}(y)=\frac{y}{l}$.

Let us remark that, since the analytical solution belongs to the finite element space, this example allows to quantify the error associated to the mortar element method. In Figure 5 are reported the spatial errors when the numerical solution $u_{h}$ is computed on differently meshes that in one case match and in the other do not match at the interface. These errors are given by expression (86) in which the temporal variable has been neglected. Looking to Figure 5, we note that, in the logarithm scale, the spatial errors depend linearly on the number of mesh nodes on $\Gamma$. Moreover, the displayed lines has angular coefficient equal to one confirming the first order of the proposed method.

In the matching case, the error should be equal to zero. Its non-zero value is due to the fact that the element of the coupling matrix $Q$ are computed by using the first approach described in Section 5.1.

(Case 2): let us consider the magnetodynamic problem described by equation (2) and we suppose that the rotor does not move. In this case, the source is represented by a sinusoidal magnetic field parallel to the $z$-axis, with intensity is $-1 \mathrm{~T} / \mathrm{m}$ the bottom and $1 \mathrm{~T} / \mathrm{m}$ at the top of the stator domain; homogeneous Neumann conditions are considered on the stator vertical boundaries. Due to these assumptions, the magnetic field $\mathbf{H}=(0,0, \mathrm{H})$ depends only on coordinates $y$ and $t$ and is solution of the following problem

$$
\left\{\begin{array}{l}
\sigma \mu \frac{\partial \mathrm{H}}{\partial t}-\frac{\partial^{2} \mathrm{H}}{\partial y^{2}}=0 \\
\mathrm{H}(l, t)=\cos (\omega t), \quad \mathrm{H}(-l, t)=-\cos (\omega t), \quad \forall t \in[0, T] .
\end{array}\right.
$$




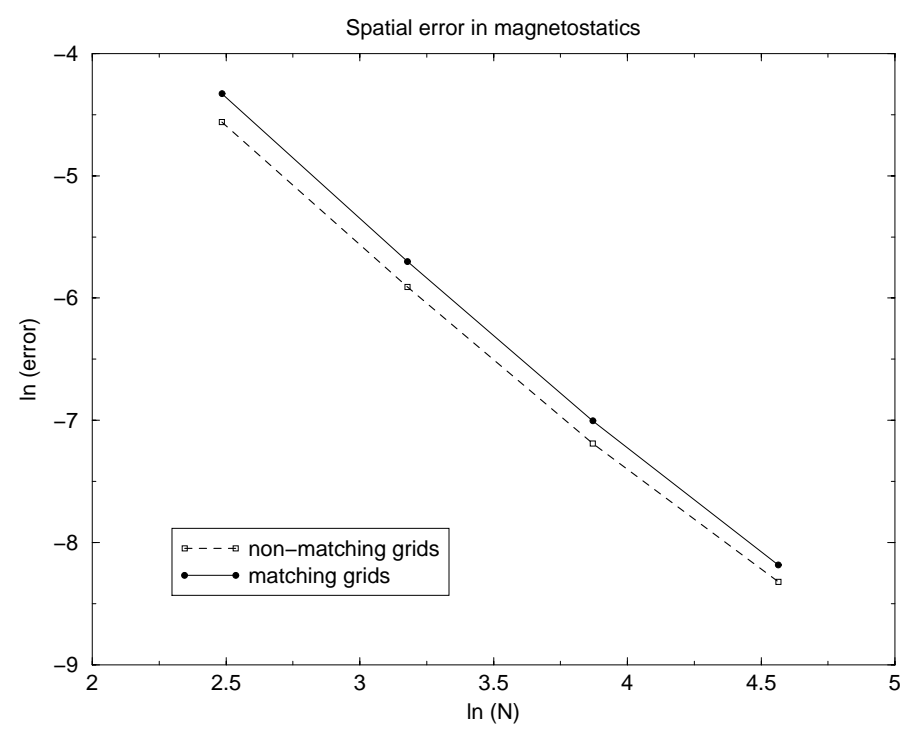

Figure 5. Spatial error (case 1): the matching and not-matching cases are compared.

In this example we have chosen the source frequency $f=1 \mathrm{~Hz}(\omega=2 \pi \mathrm{rad} / \mathrm{s})$ and the final instant $T=2 \mathrm{~s}$. The (third component of the) analytical (exact) solution of the problem is given by

$$
\mathrm{H}(y, t)=\operatorname{Real}\left\{\frac{\sinh (\lambda y)}{\sinh (\lambda l)} \mathrm{e}^{\mathrm{i} \omega t}\right\}, \quad \lambda=\frac{(1+i)}{\delta} .
$$

In Figure 6 are reported the temporal errors when the numerical solution $u_{h}$ is computed on the finest mesh with different time steps, both in the matching and non-matching cases. The considered time steps (in seconds) are

$$
\delta t=\frac{\delta t_{1}}{102^{s}} \quad \text { and } \quad s=0,1,2,3 .
$$

Since on $\Gamma$ the mesh size is small, the temporal error of the non-matching case does not significantly differ from that of the matching case. Moreover, both the errors depend linearly on the time step.

In Figure 7 are reported the spatial errors when the numerical solution $u_{h}$ is computed with the smallest time step (corresponding to $s=3$ ) on different meshes that in one case match and in the other do not match at the interface. In the logarithm scale, the spatial errors depend linearly on the number of mesh nodes on $\Gamma$.

Let us remark that, in both Figures 6 and 7, the fact that the two lines are almost parallel implies that the order of the conforming and non-conforming method is the same. In addition, it has to be noted that the constant contained in the error estimate related to the non-matching case is close to the constant contained in the error estimate related to the matching case since the two lines are very close.

(Case 3): let us consider the magnetodynamic problem described by equation (2) with the same conditions of case 1 . The exact solution is not of analytical type but computed numerically on the finest mesh with the smallest time step defined below. In this third case, the rotor will move at a constant speed. The rotation angle is chosen so that the finest rotor mesh is always matching with the stator finest one at the nodes lying on $\Gamma$. In this way, we get rid of the numerical error due to the geometric non-conformity while computing the exact solution. 


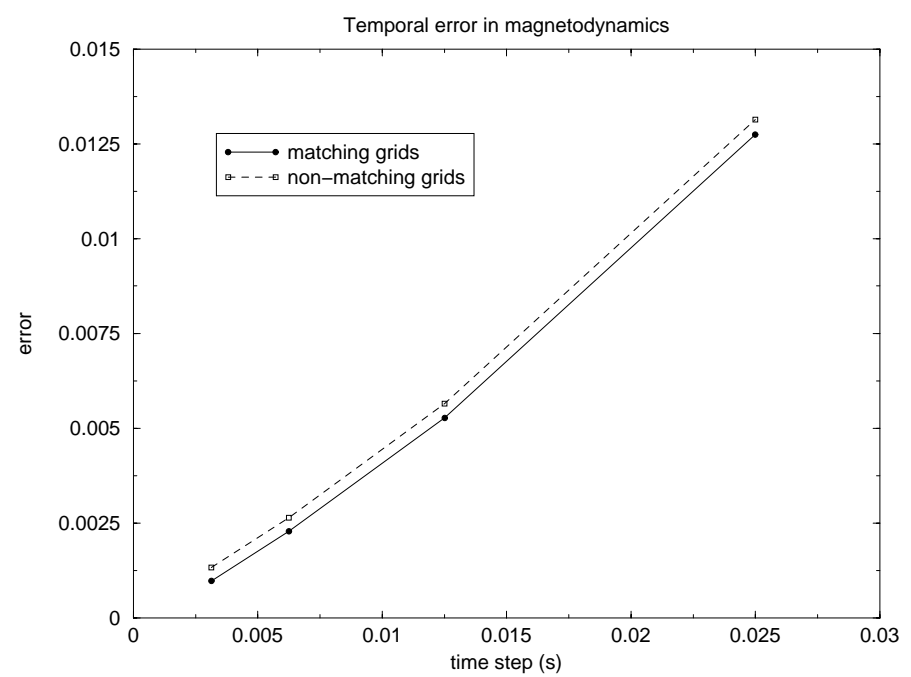

Figure 6. Temporal error computed on the finest mesh (case 2): the matching and notmatching cases are compared.

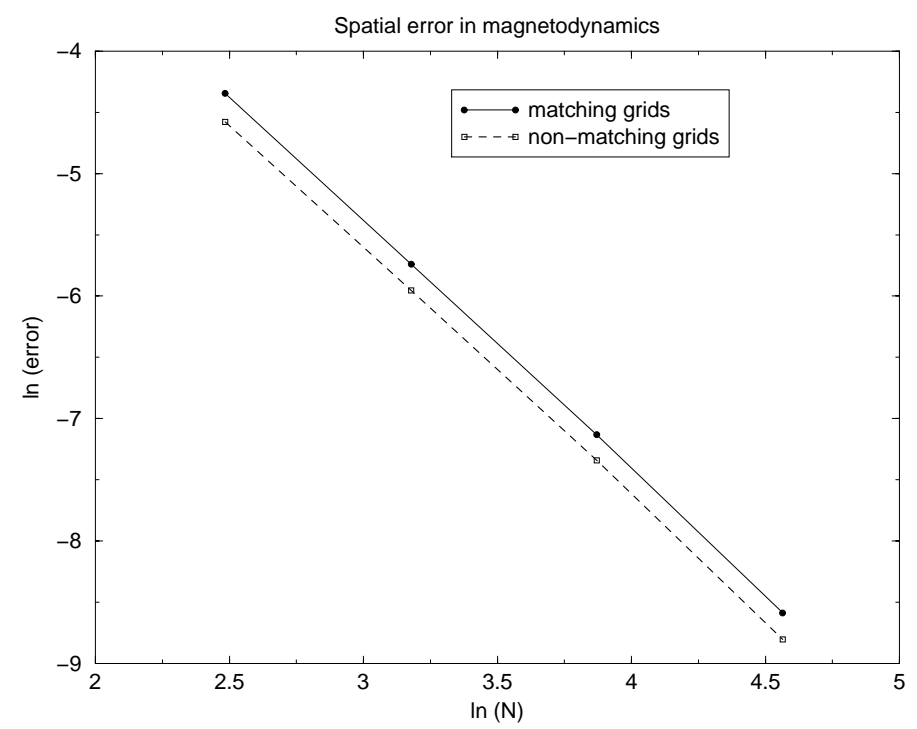

FiguRE 7. Spatial error computed with the smallest time step (case 2): the matching and not-matching cases are compared.

The computation of the spatial and temporal errors rely on different choices of the simulation parameters which are connected to the rotor movement. Since we want to analyse the influence of the rotor movement on the precision of the method, we will consider two different tangential speeds: $v^{\prime} \approx 10 v_{1}$ and $v^{\prime \prime} \approx 100 v_{1}$ with $v_{1}$ defined in Section 1. 
Spatial error parameters. During the numerical tests for the spatial error, we impose that the rotation angle at each time step is equal to $7.5^{\circ}$. This angle is chosen so that the finest rotor mesh (obtained with $r=4$ ) is, at each rotation, always matching with the finest stator one in all the nodes lying on $\Gamma$; the smallest angle that allows for this geometric conformity at $r=4$ is $360^{\circ} / 192=1.875^{\circ}$. Since we use a rotation angle that is four times bigger than $1.875^{\circ}$, the geometric conformity is also preserved for $r=2$ and $r=3$. For $r=0$ and $r=1$, instead, we loose the geometric conformity. In relation to the chosen rotation angle, the correct time step for the tangential speed $v^{\prime}$ is $\delta t=\delta t_{1} / 10 \approx 0.0025 \mathrm{~s}$ and for $v^{\prime \prime}$ it is $\delta t=\delta t_{1} / 100 \approx 0.00025 \mathrm{~s}$.

Temporal error parameters. To get the temporal error, we compute the numerical solution on the finest mesh $\left(r=4\right.$ for both domains) with different time steps. Two rotation velocities are considered, $v^{\prime}$ and $v^{\prime \prime}=10 v^{\prime}$.

Results on the spatial and temporal errors related to the chosen rotation speeds are contained in Figures 8 and 9. The time interval where the transient effects take place varies with the rotation speed and it is difficult to localize it within the simulation interval. For these reasons, we have computed the error when the steady-state configuration is achieved. Moreover, at the steady-state configuration, the distribution of the values of the magnetic field as well as of the current density does not depend on time.

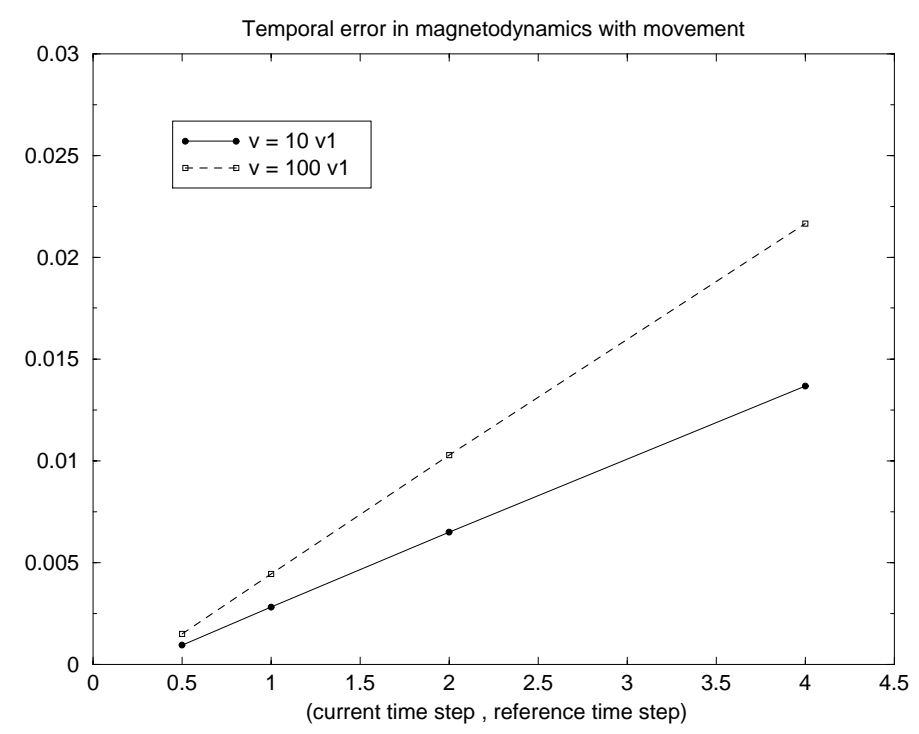

Figure 8. Temporal error computed on the finest mesh (case 3) when the rotor moves at constant speed: for $v^{\prime}$ the reference time step is $\delta t_{1} / 10$ and for $v^{\prime \prime}$ the reference time step is $\delta t_{1} / 100$.

The spatial error associated to $v^{\prime \prime}$ behaves as the spatial error related to $v^{\prime}$ : its smaller (absolute) values are due to the fact that the faster the rotor moves, the smaller the magnetic field values at the interface are (as it was observed in Fig. 4). As explained before, for $r=0$ and $r=1$ we deal with non-matching grids: despite this, in Figure 9 we can see that the corresponding error values lie on a straight line. Moreover, at the rotation speed $v^{\prime \prime}$ the constant in the error estimate related to the non-conforming case is not much bigger than that in the same error estimate at speed $v^{\prime}$.

Since for both experiments we obtain straight lines, the first order of the method is actually numerically verified.

Concerning Figure 8, the temporal errors are plotted with respect to the ratio between the current time step and the reference one (obtained with $s=0$ at both speeds). This choice comes from the wish of drawing in the same graph the errors related to different speeds which are characterized by different time step scales. In both cases, the temporal error behaves linearly with respect to the time step. 


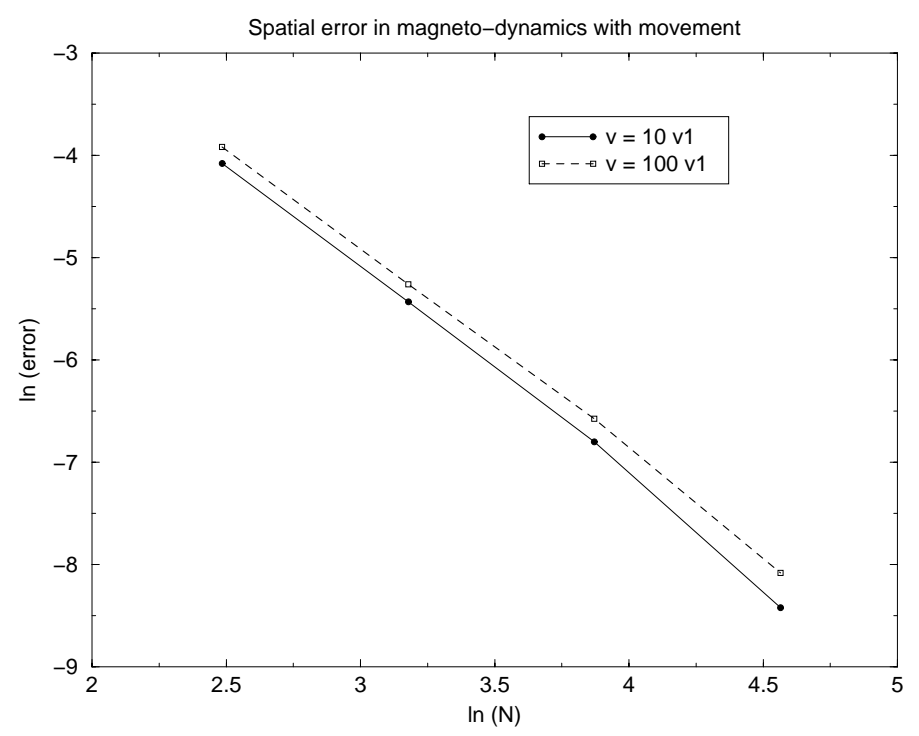

Figure 9. Spatial error computed with the smallest time step (case 3): the rotor moves at two different constant angular speeds.

\subsection{A realistic simulation}

In the previous section the physical interpretation of the numerical results was not the point. The tests have been done in order to give qualitative and quantitative "support" to the theory developed at the beginning of the paper. The aim of this section is to use the mortar tool to analyse a more realistic situation.

The simulation of the induced currents propagation into the domain represented in Figure 2 (left), is characterized by two intervals: a first one during which the rotor is kept fixed and the magnetic field invades the whole domain with a constant gradient and a second one in which the rotor moves around its axis. Both intervals are important and the simulation parameters have to be chosen in such a way that these periods are respected. Taking care of this aspect, we can analyse the effect of the rotor movement on the established magnetic field distribution, avoiding wrong interpretations of the numerical results. Another important point is to understand what means to accelerate the rotor movement. The acceleration of the rotor movement is not obtained increasing the angle of which the rotor turns at each time step but decreasing the time step itself. This is more natural as regard the error bound.

The numerical results displayed in Figure 10 are obtained in the first case (left) by considering nit $_{1}=800$ time iterations with time step of length $\delta t^{\prime}=0.2510^{-3} \mathrm{~s}$ and keeping the rotor rotating with speed $v^{\prime}=0.6310^{3}$ $\mathrm{rad} / \mathrm{s}$. In the second case (right) we accomplish nit $_{2}=8000$ time iterations $\left(\right.$ nit $_{2}=10$ nit $\left._{1}\right)$ with time step of length $\delta t^{\prime \prime}=0.2510^{-4} \mathrm{~s}\left(\delta t_{2}=\delta t_{1} / 10\right)$ and the rotor is rotating with speed $v^{\prime \prime}=0.6310^{4} \mathrm{rad} / \mathrm{s}\left(v^{\prime \prime}=10 v^{\prime}\right)$. We have then that the rotor moves of $9^{\circ}$ in the first case and of $90^{\circ}$ in the second one, each time step $\delta t^{\prime}$.

In Figure 10 can be seen the effects of the rotor movement on the current density distribution: each arrow represents the current density vector at an element barycenter. Looking at Figure 10, we remark that the faster the rotor moves, the more the currents circulate in the stator domain while in the rotor domain they concentrate near the sliding interface forming a limit layer.

Moreover, in the rotor domain, the current density distribution is such to create an angular momentum that contrasts the rotation: this contrasting effect increases with the rotation speed.

At the web address http://www.asci.fr/Francesca.Rapetti/figures.html is available an animation that reproduces at each time step the computed magnetic field distribution, starting from an linear one. When the rotor starts moving, a transient phenomenon takes place; soon after, the transient solution lets the way to a 

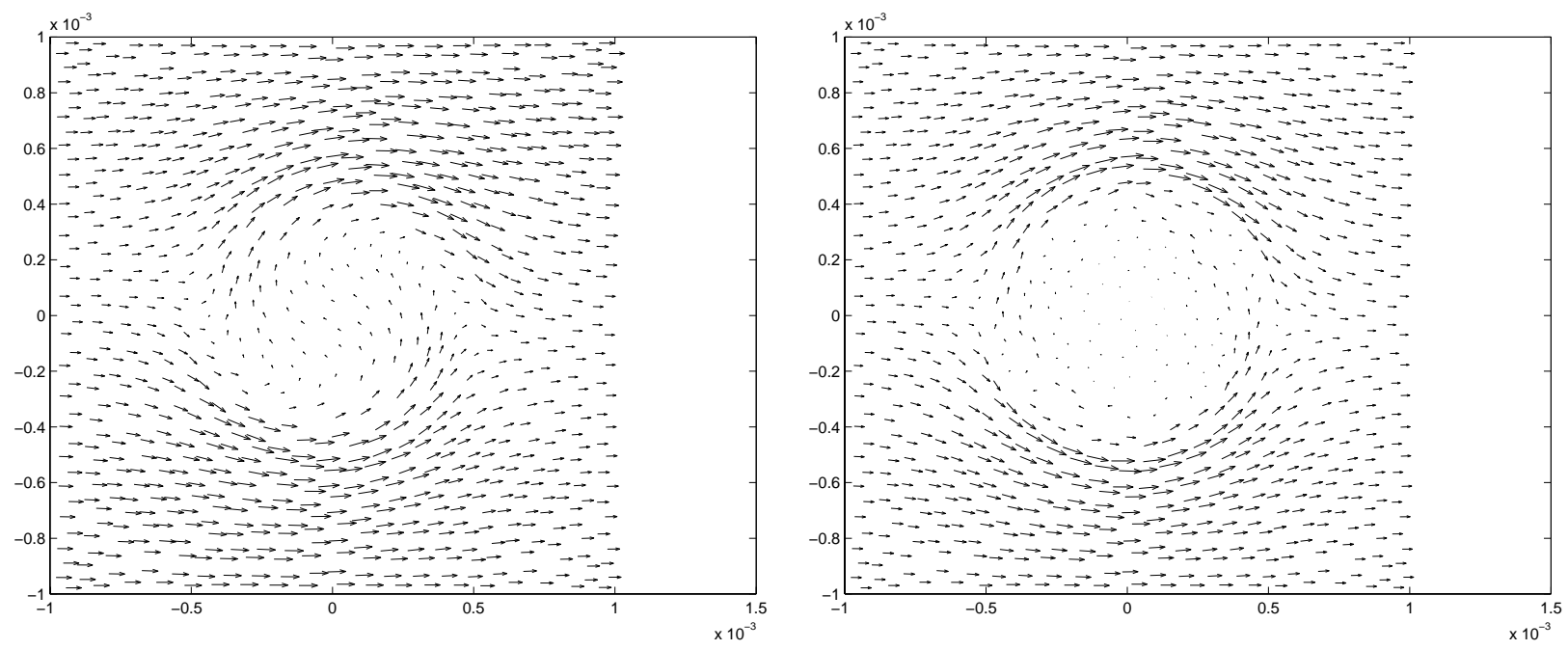

FiguRE 10. Effect of the rotor movement on the current density distribution. Steady state configurations with the rotor moving at $v^{\prime}=0.6310^{3} \mathrm{rad} / \mathrm{s}$ (left) and $v^{\prime \prime}=10 v^{\prime}$ (right).

steady-state one. We can see a similarity between this problem and the heat one when the solution is computed in a domain with a moving part.

Further results on the influence of the rotor movement on the currents distribution as well as on the power losses in dependence of the rotor angular speed are presented in [24].

Acknowledgements. The authors thank Prof. Alain Bossavit, Prof. Frederic Bouillault and Prof. Adel Razek for their disponibility and helpful suggestions. This work has the financial support of the European Community (TMR Contract number ERB4001GT965424), of I.A.N.-C.N.R. (Italy) and of C.N.R.S. (France).

\section{REFERENCES}

[1] R. Adams, Sobolev spaces. Academic Press, London (1976).

[2] R. Albanese and G. Rubinacci, Formulation of the eddy-current problem. IEEE proceedings 137 (1990).

[3] G. Anagnostou, A. Patera and Y. Maday, A sliding mesh for partial differential equations in nonstationary geometries: application to the incompressible Navier-Stockes equations. Tech. rep., Laboratoire d'Analyse Numérique, Université Pierre et Marie Curie (1994).

[4] F. Ben Belgacem and Y. Maday, Non-conforming spectral element methodology tuned to parallel implementation. Comput. Meth. Appl. Mech. Engrg. 116 (1994) 59-67.

[5] F. Ben Belgacem, Y. Maday, The mortar element method for three dimensional finite elements. RAIRO-Modél. Math. Anal. Numér. 2 (1997) 289-302.

[6] C. Bernardi, Optimal finite element interpolation of curved domains. SIAM J. Numer. Anal. 26 (1989) 1212-1240.

[7] C. Bernardi, Y. Maday and A.T. Patera, A new nonconforming approach to domain decomposition: The mortar elements method, in Nonlinear partial differential equations and their applications, H. Brezis and J. Lions, Eds., Collège de France Seminar, Paris, Vol. XI (1994) 13-51.

[8] A. Bossavit, Électromagnétisme en vue de la modélisation, Springer-Verlag, Paris (1986).

[9] A. Bossavit, Calcul des courants induits et des forces électromagnétiques dans un système de conducteurs mobiles. RAIROModél. Math. Anal. Numér. 23 (1989) 235-259.

[10] A. Bossavit, Le calcul des courants de Foucault en dimension 3, avec le champ électrique comme inconnue. I: Principes. Rev. Phys. Appl. 25 (1990) 189-197.

[11] F. Bouillault, Z. Ren and A. Razek, Modélisation tridimensionnelle des courants de Foucault à l'aide de méthodes mixtes avec différentes formulations. Rev. Phys. Appl. 25 (1990) 583-592.

[12] C.J. Carpenter, Comparison of alternative formulations of 3-dimensional magnetic-field and eddy-current problems at power frequencies. IEEE proceedings 124 (1977) 1026-1034. 
[13] P. Ciarlet, The finite element method for elliptic problems. North-Holland, Amsterdam (1978).

[14] R. Dautray and J.L. Lions, Analyse mathématique et calcul numérique pour les sciences et les techniques, 2nd edn. Masson, Paris (1987)

[15] B. Davat, Z. Ren and M. Lajoie-Mazenc, The movement in field modeling. IEEE, Trans. Magn. 21 (1985) $2296-2298$.

[16] C.R.I. Emson, C.P. Riley, D.A. Walsh, K. Ueda and T. Kumano, Modeling eddy currents induced by rotating systems. IEEE, Trans. Magn. 34 (1998) 2593-2596.

[17] Y. Goldman, P. Joly and M. Kern, The electric field in the conductive half-space as a model in mining and petroleum prospection. Math. Meth. Appl. Sci. 11 (1989) 373-401.

[18] J. Jackson, Classical electrodynamics. Wiley, New York (1952).

[19] S. Kurz, J. Fetzer, G. Lehenr, and W. Rucker, A novel formulation for 3d eddy current problems with moving bodies using a Lagrangian description and bem-fem coupling. IEEE, Trans. Magn. 34 (1998) 3068-3073.

[20] R. Leis, Initial Boundary value problems in mathematical physics. John Wiley and Sons (1986).

[21] Y. Marechal, G. Meunier, J. Coulomb and H. Magnin, A general purpose for restoring inter-element continuity. IEEE, Trans. Magn. 28 (1992) 1728-1731.

[22] A. Nicolet, F. Delincé, A. Genon and W. Legros, Finite elements-boundary elements coupling for the movement modeling in two dimensional structures. J. Phys. III 2 (1992) 2035-2044.

[23] A. Quarteroni and A. Valli, Numerical approximation of partial differential equations. Ser. Comput. Math. 23, Springer-Verlag (1993).

[24] F. Rapetti, L. Santandrea, F. Bouillault and A. Razek, Simulating eddy currents distributions by a finite element method on moving non-matching grids. COMPEL 19 (2000) 10-29.

[25] A. Razek, J. Coulomb, M. Felliachi and J. Sobonnadière, Conception of an air-gap element for dynamic analysis of the electromagnetic fields in electric machines. IEEE, Trans. Magn. 18 (1982) 655-659.

[26] D. Rodger, H. Lai and P. Leonard, Coupled elements for problems involving movement. IEEE, Trans. Magn. 26 (1990) $548-550$.

[27] V. Thomeé, Galerkin finite element methods for parabolic problems. Ser. Comput. Math. 25, Springer (1997).

To access this journal online:

www.edpsciences.org 